\title{
ANALYTICAL VALIDATION OF A CONTINUUM MODEL FOR EPITAXIAL GROWTH WITH ELASTICITY ON VICINAL SURFACES
}

\author{
G. DAL MASO, I. FONSECA, AND G. LEONI
}

\begin{abstract}
In this paper it is shown existence of weak solutions of a variational inequality derived from the continuum model introduced by Xiang [7, formula (3.62)] (see also the work of Xiang and E [8] and Xu and Xiang [9]) to describe the self-organization of terraces and steps driven by misfit elasticity between a film and a substrate in heteroepitaxial growth. This model is obtained as a continuum limit of discrete theories of Duport, Politi, and Villain [3] and Tersoff, Phang, Zhang, and Lagally[6].
\end{abstract}

Keywords: singular nonlinear parabolic equations, Hilbert transform, thin films 2010 Mathematics Subject Classification: 35K55, 74K35, 44A15.

\section{CONTENTS}

1. Introduction

2. Preliminaries

3. A Notion of Weak Solution

4. Bounded Monotone Problems 8

5. An Auxiliary Problem 12

6. Proof of the Main Theorems 19

Acknowledgments 20

References 20

\section{INTRODUCTION}

In this paper we prove existence of weak solutions of a variational inequality derived from the continuum model introduced by Xiang [7] (see also the work of Xiang and E [8] and $\mathrm{Xu}$ and Xiang [9]) to describe the self-organization of terraces and steps driven by misfit elasticity between a film and a substrate in heteroepitaxial growth. This model is obtained as a continuum limit of discrete theories of Duport, Politi, and Villain [3] and Tersoff, Phang, Zhang, and Lagally[6].

The evolution equation derived by Xiang in [7, formula (3.62)] is

$$
\dot{h}=\left[-H\left(h_{x}\right)-\left(\frac{1}{h_{x}}+h_{x}\right) h_{x x}\right]_{x x},
$$

where $\dot{h}$ denotes the derivative of $h$ with respect to $t$. Here, the function $h$ describes the height of the surface of the film, and it is assumed to be monotone. Without loss of generality, we take $h$ to be increasing. Note that in [7] and [8] $h$ is taken to be decreasing with respect to $x$, therefore in those papers $h(t, x)$ corresponds to our $h(t,-x)$. Moreover, $H$ denotes the periodic Hilbert transform (see (2.25) below). 
To exploit the variational structure of equation (1.1), we consider the function $\Phi: \mathbb{R} \rightarrow$ $\mathbb{R} \cup\{+\infty\}$ introduced in [7, formula (3.64)] and defined by

$$
\Phi(\xi):= \begin{cases}\xi \log \xi+\frac{1}{6} \xi^{3} & \text { if } \xi>0, \\ 0 & \text { if } \xi=0, \\ +\infty & \text { otherwise. }\end{cases}
$$

Note that $\Phi$ is convex, and that (1.1) can be written as

$$
\dot{h}=\left[-H\left(h_{x}\right)-\left(\Phi^{\prime}\left(h_{x}\right)\right)_{x}\right]_{x x},
$$

where the derivative $\Phi^{\prime}$ is given by

$$
\Phi^{\prime}(\xi)=\log \xi+1+\frac{1}{2} \xi^{2} \quad \text { if } \xi>0 .
$$

In Theorem 3.1 we show that existence of solutions of (1.3) with $h_{x}$ bounded away from zero is equivalent to the existence of solutions of the parabolic evolution equation

$$
\dot{u}=-\left[H\left(u_{x x}\right)\right]_{x}-\left[\Phi_{a}^{\prime}\left(u_{x x}\right)\right]_{x x},
$$

where $u$ is an appropriate anti-derivative of $h, a$ is a positive constant, and

$$
\Phi_{a}(\xi):=\Phi(\xi+a) .
$$

We study equation (1.5) on a time interval $[0, T]$, for some $T>0$, and on the space interval $I:=(-\pi, \pi)$, with inital boundary condition at $t=0$ and periodic boundary conditions on $\partial I$. In this work we use the spaces $L_{\text {per }_{0}}^{2}(I)$ and $W_{\text {per }_{0}}^{2,3}(I)$ of $2 \pi$-periodic functions of $L_{\mathrm{loc}}^{2}(\mathbb{R})$ and $W_{\text {loc }}^{2,3}(\mathbb{R})$, respectively, with average 0 on $I$. The main difficulty in the analysis of (1.5) is due to the singularity of $\log \xi$ in (1.4) at the origin. To circumvent this problem, we will consider a family of approximating problems (see (5.1) below), and we will prove that their solutions converge to a solution of the variational inequality (1.7). The central result of his paper is the following theorem.

Theorem 1.1. Let $a>0$ and let $u^{0} \in L_{\text {per }_{0}}^{2}(I)$. Then there exists $u \in L^{3}\left([0, T] ; W_{\text {per }_{0}}^{2,3}(I)\right)$ such that

$$
\begin{aligned}
& \int_{0}^{T}\left(\langle\dot{w}(t), w(t)-u(t)\rangle_{\left(W_{\text {per }_{0}}^{2,3}(I)\right)^{\prime}, W_{\text {per }_{0}}^{2,3}(I)}+\mathcal{F}_{a}(w(t))\right) d t \\
& \quad \geq \int_{0}^{T}\left(\mathcal{F}_{a}(u(t))+\langle\mathcal{H}(u(t)), w(t)-u(t)\rangle_{\left(W_{\text {per }_{0}}^{2,3}(I)\right)^{\prime}, W_{\text {per }_{0}}^{2,3}(I)}\right) d t
\end{aligned}
$$

for every $w \in L^{3}\left([0, T] ; W_{\text {per }_{0}}^{2,3}(I)\right)$, with $\dot{w} \in L^{3 / 2}\left([0, T] ;\left(W_{\text {per }_{0}}^{2,3}(I)\right)^{\prime}\right)$ and $w(0)=u^{0}$.

Here, the functional $\mathcal{F}_{a}: W_{\text {per }_{0}}^{2,3}(I) \rightarrow \mathbb{R} \cup\{+\infty\}$ is defined by

$$
\mathcal{F}_{a}(u):=\int_{I} \Phi_{a}\left(u_{x x}\right) d x,
$$

and $\mathcal{H}: W_{\text {per }_{0}}^{2,3}(I) \rightarrow\left(W_{\text {per }_{0}}^{2,3}(I)\right)^{\prime}$ is the operator given by

$$
\langle\mathcal{H}(u), v\rangle_{\left(W_{\text {per }_{0}}^{2,3}(I)\right)^{\prime}, W_{\text {per }_{0}}^{2,3}(I)}:=\int_{I} H\left(u_{x x}\right) v_{x} d x .
$$

Note that in Proposition 3.4 we prove that strong solutions of (1.5) with $u_{x x}+a$ bounded away from zero satisfy (1.7), so that this variational inequality can be considered as a weak formulation of (1.5).

Let $\mathcal{A}_{a}: D_{a} \rightarrow\left(W^{2, \infty}(I)\right)^{\prime}$ be the operator defined by

$$
\left\langle\mathcal{A}_{a}(u), v\right\rangle_{\left(W^{2, \infty}(I)\right)^{\prime}, W^{2, \infty}(I)}:=\int_{I} \Phi_{a}^{\prime}\left(u_{x x}\right) v_{x x} d x,
$$

where

$$
D_{a}:=\left\{u \in W^{2,2}(I): \log \left(u_{x x}+a\right) \in L^{1}(I)\right\} .
$$


We prove also the following result, where the variational inequality is more similar to equation (1.5).

Theorem 1.2. Under the assumptions of Theorem 1.1, the solution $u$ of problem (1.7) satisfies $\log \left(u_{x x}+a\right) \in L^{1}\left([0, T] ; L^{1}(I)\right)$, and

$$
\begin{gathered}
\int_{0}^{T}\left(\langle\dot{w}(t), w(t)-u(t)\rangle_{\left(W_{\text {pero }_{0}}^{2,3}(I)\right)^{\prime}, W_{\text {pero }_{0}}^{2,3}(I)}+\left\langle\mathcal{A}_{a}(u(t)), w(t)-u(t)\right\rangle_{\left(W^{2, \infty}(I)\right)^{\prime}, W^{2, \infty}(I)}\right) d t \\
\geq \int_{0}^{T}\langle\mathcal{H}(u(t)), w(t)-u(t)\rangle_{\left(W_{\text {per }_{0}}^{2,3}(I)\right)^{\prime}, W_{\text {per }_{0}}^{2,3}(I)} d t
\end{gathered}
$$

for every $w \in L^{3}\left([0, T] ; W_{\text {per }_{0}}^{2,3}(I)\right)$, with $\dot{w} \in L^{3 / 2}\left([0, T] ;\left(W_{\text {per }_{0}}^{2,3}(I)\right)^{\prime}\right)$ and $w(0)=u^{0}$, such that $w-u \in L^{\infty}\left([0, T] ; W^{2, \infty}(I)\right)$ and $\log \left(w_{x x}+a\right) \in L^{1}\left([0, T] ; L^{1}(I)\right)$.

\section{Preliminaries}

We begin with a compactness result on Banach spaces, which extends [5, Chapter 1,Theorem 5.1] to the case of $L^{1}\left([0, T] ; B_{1}\right)$, without assuming that $B_{1}$ is reflexive.

Theorem 2.1. Let $B_{0}, B$, and $B_{1}$ be Banach spaces, and let $1<p<+\infty$. Assume that

$$
\begin{aligned}
& B_{0} \hookrightarrow B \hookrightarrow B_{1} \text { with continuous embeddings, } \\
& B_{0} \text { is reflexive, } \\
& \text { the embedding } B_{0} \hookrightarrow B \text { is compact. }
\end{aligned}
$$

Let $\mathcal{V}$ be the Banach space of all functions $v \in L^{p}\left([0, T] ; B_{0}\right)$ whose distributional derivative $\dot{v}$ belongs to $L^{1}\left([0, T] ; B_{1}\right)$ endowed with the norm

$$
\|v\|_{\mathcal{V}}:=\|v\|_{L^{p}\left([0, T] ; B_{0}\right)}+\|\dot{v}\|_{L^{1}\left([0, T] ; B_{1}\right)} .
$$

Then the embedding $\mathcal{V} \hookrightarrow L^{p}([0, T] ; B)$ is compact.

Proof. Let $\left\{v_{n}\right\}$ be a bounded sequence in $\mathcal{V}$. Using (2.4) we obtain that

$$
\begin{aligned}
& \left\{v_{n}\right\} \text { is bounded in } L^{p}\left([0, T] ; B_{0}\right), \\
& \left\{\dot{v}_{n}\right\} \text { is bounded in } L^{1}\left([0, T] ; B_{1}\right) .
\end{aligned}
$$

Since $1<p<+\infty$, by $(2.2)$ the space $L^{p}\left([0, T] ; B_{0}\right)$ is reflexive (see, e.g., [4, Theorem $2.112])$. By (2.5), extracting a subsequence (not relabeled), we have that

$$
v_{n} \rightarrow v \text { weakly in } L^{p}\left([0, T] ; B_{0}\right)
$$

for some $v \in L^{p}\left([0, T] ; B_{0}\right)$.

By [5, Chapter 1, Lemma 5.1], for every $\eta>0$ there exists $c_{\eta}>0$ such that

$$
\|u\|_{B} \leq \eta\|u\|_{B_{0}}+c_{\eta}\|u\|_{B_{1}}
$$

for every $u \in B_{0}$. It follows that

$$
\left\|v_{n}-v\right\|_{L^{p}([0, T] ; B)} \leq \eta\left\|v_{n}-v\right\|_{L^{p}\left([0, T] ; B_{0}\right)}+c_{\eta}\left\|v_{n}-v\right\|_{L^{p}\left([0, T] ; B_{1}\right)}
$$

for every $n$. By (2.5), for every $\varepsilon>0$ there exists $\eta>0$ such that

$$
\left\|v_{n}-v\right\|_{L^{p}([0, T] ; B)} \leq \varepsilon+c_{\eta}\left\|v_{n}-v\right\|_{L^{p}\left([0, T] ; B_{1}\right)}
$$

for every $n$. Therefore, to prove that $v_{n} \rightarrow v$ strongly in $L^{p}([0, T] ; B)$ it is enough to show that

$$
v_{n} \rightarrow v \text { strongly in } L^{p}\left([0, T] ; B_{1}\right) .
$$

Since $\mathcal{V} \hookrightarrow W^{1,1}\left((0, T) ; B_{1}\right) \hookrightarrow C^{0}\left([0, T] ; B_{1}\right)$ with continuous embedding, the sequence $\left\{v_{n}\right\}$ is bounded in $C^{0}\left([0, T] ; B_{1}\right)$. By the Dominated Convergence Theorem, to obtain (2.8) it suffices to prove that

$$
v_{n}(t) \rightarrow v(t) \text { strongly in } B_{1} \text { for a.e. } t \in[0, T] .
$$


For $t \in[0, T]$ and $n \in \mathbb{N}$ define

$$
V_{n}(t):=\int_{0}^{t}\left\|\dot{v}_{n}(s)\right\|_{B_{1}} d s
$$

By (2.6), $\left\{V_{n}\right\}$ is a bounded sequence of monotone functions. By the Helly Theorem there exists a subsequence (not relabeled) that converges pointwise to a monotone function $V:[0, T] \rightarrow[0,+\infty)$.

Let $t_{0}$ be a continuity point of $V$ and a Lebesgue point of $v$, considered as an integrable function with values in $B_{1}$, i.e.,

We want to prove that

$$
\lim _{s \rightarrow 0} \frac{1}{s} \int_{t_{0}}^{t_{0}+s}\left\|v(t)-v\left(t_{0}\right)\right\|_{B_{1}} d t=0
$$

$$
v_{n}\left(t_{0}\right) \rightarrow v\left(t_{0}\right) \text { strongly in } B_{1} .
$$

Fix $\varepsilon>0$ and $s>0$ such that

$$
V\left(t_{0}+s\right)-V\left(t_{0}\right)<\varepsilon \quad \text { and } \quad \frac{1}{s} \int_{t_{0}}^{t_{0}+s}\left\|v(t)-v\left(t_{0}\right)\right\|_{B_{1}} d t<\varepsilon .
$$

Using an argument due to R. Temam (see [5, Chapter 1, Theorem 5.1]), we write

$$
v_{n}\left(t_{0}\right)=\frac{1}{s} \int_{t_{0}}^{t_{0}+s} v_{n}(t) d t-\frac{1}{s} \int_{t_{0}}^{t_{0}+s}\left(t_{0}+s-t\right) \dot{v}_{n}(t) d t=: a_{n}-b_{n}
$$

and we define

$$
a:=\frac{1}{s} \int_{t_{0}}^{t_{0}+s} v(t) d t
$$

By (2.7), the sequence $\left\{a_{n}\right\}$ converges to $a$ weakly in $B_{0}$. By (2.1), (2.2), and (2.3), it converges strongly in $B_{1}$. Since $\left\|a-v\left(t_{0}\right)\right\|_{B_{1}}<\varepsilon$ by (2.11), we obtain

$$
\lim _{n \rightarrow \infty}\left\|a_{n}-v\left(t_{0}\right)\right\|_{B_{1}}<\varepsilon \text { for } n \text { large enough. }
$$

On the other hand,

$$
\left\|b_{n}\right\|_{B_{1}} \leq \int_{t_{0}}^{t_{0}+s}\left\|\dot{v}_{n}(t)\right\|_{B_{1}} d t=V_{n}\left(t_{0}+s\right)-V_{n}\left(t_{0}\right),
$$

so that, by (2.11),

$$
\limsup _{n \rightarrow \infty}\left\|b_{n}\right\|_{B_{1}} \leq V\left(t_{0}+s\right)-V\left(t_{0}\right)<\varepsilon .
$$

In view of the arbitrariness of $\varepsilon>0,(2.10)$ follows from (2.12), (2.13), and (2.14). Since the continuity points for $V$ that are Lebesgue points for $v$ form a set of full measure, we have proved (2.9), which concludes the proof of the theorem.

In what follows, given a Banach space $B$, we denote by $\langle\cdot, \cdot\rangle_{B^{\prime}, B}$ the duality between $B$ and its dual $B^{\prime}$.

Let $I:=(-\pi, \pi)$. To introduce the functional setting for the study of equation (1.3), for $k \in \mathbb{Z}$ and $1 \leq p<+\infty$, consider the space

$$
W_{\text {per }}^{k, p}(I):=\left\{u \in W_{\text {loc }}^{k, p}(\mathbb{R}): u \text { is } 2 \pi \text {-periodic }\right\},
$$

where the periodicity of $u$ when $k<0$ is to be understood in the sense of distributions. If $k \geq 0$ then the space $W_{\text {per }}^{k, p}(I)$ is endowed with the norm induced by $W^{k, p}(I)$. If $k<0$ and $(1 / p)+(1 / q)=1$, then $W_{\text {per }}^{k, q}(I)$ is the dual of $W_{\text {per }}^{-k, p}(I)$, and is endowed with the dual norm. For simplicity, we use the notation

$$
\langle\cdot, \cdot\rangle_{k, p}:=\langle\cdot, \cdot\rangle_{\left(W_{\mathrm{per}}^{k, p}(I)\right)^{\prime}, W_{\mathrm{per}}^{k, p}(I)} .
$$

Moreover, we also define the space

$$
W_{\text {per }_{0}}^{k, p}(I):=\left\{u \in W_{\text {per }}^{k, p}(I): u_{I}=0\right\},
$$


where $u_{I}$ is the average of $u$ in one period if $k \geq 0$, and $u_{I}:=\langle u, 1\rangle_{k, p}$ when $k<0$. If $k \geq 0$ then the space $W_{\text {per }_{0}}^{k, p}(I)$ is endowed with the norm induced by $W^{k, p}(I)$. If $k<0$ and $(1 / p)+(1 / q)=1$, then it can be shown that $W_{\text {per }_{0}}^{k, q}(I)$ is the dual of $W_{\text {per }_{0}}^{-k, p}(I)$, and it will be endowed with the dual norm. With an abuse of notation, we continue to use the symbol $\langle\cdot, \cdot\rangle_{k, p}$ to denote the corresponding duality. Finally, set

$$
\begin{aligned}
W_{\text {per* }^{*}}^{1,3}(I): & =\left\{u \in W_{\text {loc }}^{1,3}(\mathbb{R}): u_{x} \text { is } 2 \pi \text {-periodic }\right\} \\
& =\left\{u \in W_{\text {loc }}^{1,3}(\mathbb{R}): u(x+2 \pi)-u(x) \text { is constant }\right\},
\end{aligned}
$$

endowed with the norm induced by $W^{1,3}(I)$.

Let

$$
V:=W_{\operatorname{per}_{0}}^{2,3}(I) \quad \text { and } \quad Y:=L_{\text {per }_{0}}^{2}(I)
$$

where

$$
L_{\text {per }_{0}}^{2}(I):=\left\{u \in L_{\text {loc }}^{2}(\mathbb{R}): u \text { is } 2 \pi \text {-periodic and } u_{I}=0\right\} .
$$

Since every $u \in V$ has mean value zero and is periodic, it follows in particular that $u_{x}$ has also mean value 0 , and so using Poincaré-Wirtinger inequality twice, there exists a constant $\alpha>0$ such that

$$
\|u\|_{W^{2,3}(I)} \leq \alpha\left\|u_{x x}\right\|_{L^{3}(I)}
$$

for every $u \in V$. This allows us to endow $V$ with the norm

$$
\|u\|_{V}:=\left\|u_{x x}\right\|_{L^{3}(I)},
$$

which is equivalent to the norm induced by $W^{2,3}(I)$. The dual space $V^{\prime}$ coincides with $W_{\text {per }_{0}}^{-2,3 / 2}(I)$ and will be endowed with the dual norm to (2.21).

In order to study the evolution equation, we introduce the Banach space

$$
\mathcal{W}:=\left\{u: u \in L^{3}([0, T] ; V), \dot{u} \in L^{3 / 2}\left([0, T] ; V^{\prime}\right)\right\},
$$

endowed with the norm

$$
\|u\|_{\mathcal{W}}:=\|u\|_{L^{3}([0, T] ; V)}+\|\dot{u}\|_{L^{3 / 2}\left([0, T] ; V^{\prime}\right)} .
$$

It is well known that $\mathcal{W} \subset C^{0}([0, T] ; Y)$, with continuous embedding (see, e.g., [10, Proposition 23.23]).

Lemma 2.2. The embedding $i: \mathcal{W} \hookrightarrow L^{3}\left([0, T] ; W_{\text {per }_{0}}^{1,3}(I)\right)$ is compact.

Proof. It is enough to apply [5, Chapter 1 , Theorem 5.1] with $p_{0}:=3, p_{1}:=3 / 2, B_{0}:=V$, $B:=W_{\text {per }_{0}}^{1,3}(I)$, and $B_{1}:=V^{\prime}$.

Consider the adjoint embedding $i^{*}: L^{3 / 2}\left([0, T] ; W_{\text {per }_{0}}^{-1,3 / 2}(I)\right) \rightarrow \mathcal{W}^{\prime}$. By its definition, for every $f \in L^{3 / 2}\left([0, T] ; W_{\operatorname{per}_{0}}^{-1,3 / 2}(I)\right)$ and every $v \in \mathcal{W}$ we have

$$
\left\langle i^{*}(f), v\right\rangle_{\mathcal{W}^{\prime}, \mathcal{W}}=\int_{0}^{T}\langle f(t), i(v)(t)\rangle_{1,3} d t=\int_{0}^{T}\langle f(t), v(t)\rangle_{V^{\prime}, V} d t .
$$

In view of Lemma 2.2 we have the following result.

Lemma 2.3. The embedding $i^{*}: L^{3 / 2}\left([0, T] ; W_{\operatorname{per}_{0}}^{-1,3 / 2}(I)\right) \rightarrow \mathcal{W}^{\prime}$ is compact.

The periodic Hilbert transform $H(u)$ of a function $u \in L_{\mathrm{per}}^{p}(I)$ is the $2 \pi$-periodic function defined by

$$
H(u)(x):=\frac{1}{2 \pi} \mathrm{PV} \int_{-\pi}^{\pi} u(x-y) \cot \left(\frac{y}{2}\right) d y
$$

where PV denotes the Cauchy principal value.

For a proof of the following proposition, we refer to [2, Proposition 9.1.3]. 
Proposition 2.4. Let $1<p<+\infty$ and let $u \in L_{\mathrm{per}}^{p}(I)$. Then $H(u) \in L_{\mathrm{per}}^{p}(I)$ and

$$
\|H(u)\|_{L^{p}(I)} \leq C_{p}\|u\|_{L^{p}(I)}
$$

for some constant $C_{p}>0$.

Using the argument in the proof of [2, Proposition 8.3.7], where Proposition 9.3.1 is used in place of Proposition 8.3.1, we obtain the following result.

Proposition 2.5. Let $1<p<+\infty$ and let $u \in W_{\mathrm{per}}^{1, p}(I)$. Then $H(u) \in W_{\mathrm{per}}^{1, p}(I)$ and $H\left(u_{x}\right)=(H(u))_{x}$ a.e. on $I$.

Remark 2.6. In view of the latter proposition, it is possible to extend $H$ as a linear bounded operator from $W_{\text {per }}^{-1, p^{\prime}}(I)$ into itself.

\section{A Notion of Weak Solution}

In this section we derive a notion of weak solution for equation (1.3). In order to transform (1.3) into a parabolic equation, we will derive an evolution equation for an appropriate antiderivative of $h$. In what follows we use the notation (2.16).

Theorem 3.1. The following conditions are equivalent:

(i) There exists $h \in L^{3}\left([0, T] ; W_{\text {per* }}^{1,3}(I)\right)$ with $\dot{h} \in L^{3 / 2}\left([0, T] ; W_{\text {per }}^{-3,3 / 2}(I)\right)$ solution of equation (1.3) satisfying

$$
h_{x}(t, x) \geq \delta \text { for a.e. } x \in \mathbb{R} \text { and } t \in[0, T],
$$

for some constant $\delta>0$.

(ii) There exist $a>0$ and $u \in L^{3}\left([0, T] ; W_{\text {per }_{0}}^{2,3}(I)\right)$ with $\dot{u} \in L^{3 / 2}\left([0, T] ; W_{\text {per }_{0}}^{-2,3}(I)\right)$ solution of

satisfying

$$
\dot{u}=-\left[H\left(u_{x x}\right)\right]_{x}-\left[\Phi_{a}^{\prime}\left(u_{x x}\right)\right]_{x x}
$$

$$
u_{x x}(t, x) \geq-a+\delta \text { for a.e. } x \in \mathbb{R} \text { and } t \in[0, T] .
$$

Observe that, in view of (1.4), the expression $\left[-H\left(h_{x}\right)-\Phi^{\prime}\left(h_{x}\right)_{x}\right]_{x x}$ belongs to the space $L^{3 / 2}\left([0, T] ; W_{\text {per }}^{-3,3 / 2}(I)\right)$, so that the equality in $(1.3)$ is well defined, i.e., for a.e. $t \in[0, T]$ we have

$$
\langle\dot{h}, \varphi\rangle_{3,3}=\left\langle-H\left(h_{x}\right)-\left(\Phi^{\prime}\left(h_{x}\right)\right)_{x}, \varphi_{x x}\right\rangle_{1,3}
$$

for every $\varphi \in W_{\text {per }}^{3,3}(I)$. Similarly, by (3.3) equation (3.2) is well-defined.

The proof of Theorem 3.1 will use the two lemmas below.

Lemma 3.2. The following conditions are equivalent:

(a) $h \in L^{3}\left([0, T] ; W_{\text {per* }}^{1,3}(I)\right)$ and $\dot{h} \in L^{3 / 2}\left([0, T] ; W_{\text {per }}^{-3,3 / 2}(I)\right)$;

(b) there exist $a \in \mathbb{R}$ and $h^{a} \in L^{3}\left([0, T] ; W_{\text {per }}^{1,3}(I)\right)$, with $\dot{h}^{a} \in L^{3 / 2}\left([0, T] ; W_{\text {per }}^{-3,3 / 2}(I)\right)$, such that $h(t, x)=h^{a}(t, x)+$ ax for a.e. $t \in[0, T]$ and every $x \in \mathbb{R}$.

Proof. The implication (b) $\Rightarrow$ (a) is straightforward. We prove that (a) implies (b). Assume that $h$ satisfies (a) and, for a.e. $t \in[0, T]$, let

$$
a(t):=\frac{1}{2 \pi} \int_{I} h_{x}(t, x) d x .
$$

If $h \in C_{c}^{\infty}\left((0, T) ; C_{\text {per* }}^{\infty}(I)\right)$ with $\dot{h} \in C_{c}^{\infty}\left((0, T) ; C_{\mathrm{per}}^{\infty}(I)\right)$, then

$$
\dot{a}(t)=\frac{1}{2 \pi} \int_{I}(\dot{h})_{x}(t, x) d x=0
$$

by the periodicity of $\dot{h}(t, \cdot)$ for every $t$. It now follows by density that $a$ is constant. Hence, $h^{a}(t, x):=h(t, x)-a x$ satisfies (b). 
Recall that the function $\Phi_{a}$ is defined in (1.6).

Lemma 3.3. Let $a>0$ and let $h^{a} \in L^{3}\left([0, T] ; W_{\text {per }}^{1,3}(I)\right)$, with $\dot{h}^{a} \in L^{3 / 2}\left([0, T] ; W_{\text {per }}^{-3,3 / 2}(I)\right)$, be a solution of

$$
\dot{h}^{a}=\left[-H\left(h_{x}^{a}\right)-\left(\Phi_{a}^{\prime}\left(h_{x}^{a}\right)\right)_{x}\right]_{x x}
$$

satisfying

$$
h_{x}^{a}(t, x) \geq-a+\delta \text { for a.e. } x \in \mathbb{R} \text { and } t \in[0, T]
$$

for some $\delta>0$. Then there exists a constant $b$, depending on the solution $h^{a}$, such that

$$
\frac{1}{2 \pi} \int_{I} h^{a}(t, x) d x=b \quad \text { for a.e. } t \in[0, T] .
$$

Proof. Let

$$
b(t):=\frac{1}{2 \pi} \int_{I} h^{a}(t, x) d x,
$$

for a.e. $t \in[0, T]$. We want to show that $b$ is constant. By $(3.8)$, we have $b(t)=$ $\left\langle h^{a}(t), 1 /(2 \pi)\right\rangle_{1,3}$, so that its distributional derivative satisfies $\dot{b}(t)=\left\langle\dot{h}^{a}(t), 1 /(2 \pi)\right\rangle_{3,3}$. Therefore (3.5) yields

$$
\begin{aligned}
\dot{b}(t) & =\left\langle\left[-H\left(h_{x}^{a}\right)-\left(\Phi_{a}^{\prime}\left(h_{x}^{a}\right)\right)_{x}\right]_{x x}, 1 /(2 \pi)\right\rangle_{3,3} \\
& =\left\langle\left[-H\left(h_{x}^{a}\right)-\left(\Phi_{a}^{\prime}\left(h_{x}^{a}\right)\right)_{x}\right]_{x}, 0\right\rangle_{2,3}=0 .
\end{aligned}
$$

We now turn to the proof of Theorem 3.1.

Proof of Theorem 3.1. Assume (i). Let $h^{a}$ be the function given by Lemma 3.2. Since $H(a)=0$ it follows that $h^{a}$ is a solution of $(3.5)$. Moreover, using the fact that $h^{a}(t, \cdot)$ is $2 \pi$-periodic, we have that the mean value of $h_{x}^{a}(t, \cdot)$ on a period is 0 , so that $(3.6)$ implies $a>0$. Define

$$
u(t, x):=\int_{-\pi}^{x}\left(h^{a}(t, y)-b\right) d y,
$$

where $b$ is the number given in Lemma 3.3. Then $x \mapsto u(t, x)$ is $2 \pi$-periodic and has mean value zero for a.e. $t \in[0, T]$. Moreover, $u_{x}=h^{a}-b, u_{x x}=h_{x}^{a}$, and $\dot{u}_{x}=\dot{h}^{a}$. It follows that $u \in L^{3}\left([0, T] ; W_{\text {per }_{0}}^{2,3}(I)\right)$ and $\dot{u} \in L^{3 / 2}\left([0, T] ; W_{\text {per }_{0}}^{-2,3 / 2}(I)\right)$. In turn, equation (3.5) can be written as

$$
\dot{u}_{x}=\left[-H\left(u_{x x}\right)-\left(\Phi_{a}^{\prime}\left(u_{x x}\right)\right)_{x}\right]_{x x} .
$$

Hence, for a.e. $t \in[0, T]$ there exists a constant $c(t) \in \mathbb{R}$ such that

$$
\dot{u}=-\left[H\left(u_{x x}\right)\right]_{x}-\left[\Phi_{a}^{\prime}\left(u_{x x}\right)\right]_{x x}+c(t),
$$

that is, for a.e. $t \in[0, T]$ we have

$$
\begin{aligned}
\frac{d}{d t}\langle u(t), \varphi\rangle_{0,3} & =\langle\dot{u}(t), \varphi\rangle_{2,3} \\
& =\left\langle H\left(u_{x x}\right), \varphi_{x}\right\rangle_{0,3}-\left\langle\Phi_{a}^{\prime}\left(u_{x x}\right), \varphi_{x x}\right\rangle_{0,3}+\langle c(t), \varphi\rangle_{0,3}
\end{aligned}
$$

for every test function $\varphi \in W_{\text {per }}^{2,3}(I)$. Taking $\varphi=1$ we get $c(t)=0$ for a.e. $t \in[0, T]$.

Therefore $u$ satisfies the equation (3.2) in the sense that, for a.e. $t \in[0, T]$, we have

$$
\langle\dot{u}(t), \varphi\rangle_{2,3}=\left\langle H\left(u_{x x}\right), \varphi_{x}\right\rangle_{0,3}-\left\langle\Phi_{a}^{\prime}\left(u_{x x}\right), \varphi_{x x}\right\rangle_{0,3}
$$

for every test function $\varphi \in W_{\text {per }}^{2,3}(I)$.

Conversely, assume (ii). Then the function $h(t, x):=u_{x}(t, x)+a x$ satisfies (i).

In this paper we establish existence and uniqueness of a solution of a variational inequality satisfied by all solutions considered in Theorem 3.1(ii). Precisely, we have the following result. 
Proposition 3.4. Let $u^{0} \in L_{\text {per }_{0}}^{2}(I)$ and let $u$ satisfy (ii) in Theorem 3.1 with $u(0)=u^{0}$. Then

$$
\log \left(u_{x x}+a\right) \in L^{3 / 2}\left([0, T] ; L^{3 / 2}(I)\right)
$$

and

$$
\begin{aligned}
& \int_{0}^{T}\left(\langle\dot{w}(t), w(t)-u(t)\rangle_{V^{\prime}, V}+\mathcal{F}_{a}(w(t))\right) d t \\
& \quad \geq \int_{0}^{T}\left(\mathcal{F}_{a}(u(t))+\langle\mathcal{H}(u(t)), w(t)-u(t)\rangle_{V^{\prime}, V}\right) d t
\end{aligned}
$$

for every $w \in \mathcal{W}$ with $w(0)=u^{0}$, where $\mathcal{F}_{a}$ is defined in (1.8).

Proof. Since $u_{x x} \in L^{3}\left([0, T] ; L^{3}(I)\right)$ and (3.3) holds, we obtain (3.10). By (1.4), this implies that $\Phi_{a}^{\prime}\left(u_{x x}\right) \in L^{3 / 2}\left([0, T] ; L^{3 / 2}(I)\right)$. Let $w$ be as in the statement. For a.e. $t \in[0, T]$ we multiply (3.2) by $w(t)-u(t)$ and add $\langle\dot{w}(t), w(t)-u(t)\rangle_{V^{\prime}, V}$ to both sides to obtain

$$
\begin{aligned}
\langle\dot{w}(t) & , w(t)-u(t)\rangle_{V^{\prime}, V}+\left\langle\Phi_{a}^{\prime}\left(u_{x x}(t)\right), w_{x x}(t)-u_{x x}(t)\right\rangle_{0,3} \\
& =\langle\dot{w}(t)-\dot{u}(t), w(t)-u(t)\rangle_{V^{\prime}, V}+\langle\mathcal{H}(u(t)), w(t)-u(t)\rangle_{V^{\prime}, V} .
\end{aligned}
$$

Since $\Phi_{a}$ is convex, we have $\mathcal{F}_{a}(w(t))-\mathcal{F}_{a}(u(t)) \geq\left\langle\Phi_{a}^{\prime}\left(u_{x x}(t)\right), w_{x x}(t)-u_{x x}(t)\right\rangle_{0,3}$. Therefore

$$
\begin{aligned}
& \int_{0}^{T}\left(\langle\dot{w}(t), w(t)-u(t)\rangle_{V^{\prime}, V}+\mathcal{F}_{a}(w(t))-\mathcal{F}_{a}(u(t))\right) d t \\
& \quad \geq \int_{0}^{T} \frac{1}{2} \frac{d}{d t}\|w(t)-u(t)\|_{L^{2}(I)}^{2} d t+\int_{0}^{T}\langle\mathcal{H}(u(t)), w(t)-u(t)\rangle_{V^{\prime}, V} d t \\
& \quad=\frac{1}{2}\|w(T)-u(T)\|_{L^{2}(I)}^{2}+\int_{0}^{T}\langle\mathcal{H}(u(t)), w(t)-u(t)\rangle_{V^{\prime}, V} d t \\
& \quad \geq \int_{0}^{T}\langle\mathcal{H}(u(t)), w(t)-u(t)\rangle_{V^{\prime}, V} d t
\end{aligned}
$$

where we used [10, Proposition 23.23] and the fact that $w(0)=u(0)=u^{0}$.

\section{Bounded Monotone Problems}

In order to overcome the difficulties due to the fact that $\Phi_{a}$ takes infinite values, we consider a suitable finite valued approximation, denoted by $\Phi_{a, \delta}$. Let

$$
\Psi(\xi):= \begin{cases}\xi \log \xi & \text { if } \xi>0 \\ 0 & \text { if } \xi=0 \\ +\infty & \text { otherwise }\end{cases}
$$

For $a \geq 0$ and $0<\delta<1 / e$ we define

$$
\Psi_{a}(\xi):=\Psi(\xi+a), \quad \Psi_{a, \delta}(\xi):= \begin{cases}(\xi+a) \log (\xi+a) & \text { if } \xi \geq-a+\delta \\ (\xi+a) \log \delta+\xi+a-\delta & \text { if } \xi \leq-a+\delta\end{cases}
$$

and

$$
\Phi_{a, \delta}(\xi):=\Psi_{a, \delta}(\xi)+\frac{1}{6}|\xi+a|^{3} .
$$

Note that $\Phi_{a, \delta}: \mathbb{R} \rightarrow \mathbb{R}$ is a convex function. In the following lemma we give some estimates on $\Phi_{a, \delta}^{\prime}$, which is given by

$$
\Phi_{a, \delta}^{\prime}(\xi)= \begin{cases}\log (\xi+a)+1+\frac{1}{2}|\xi+a|(\xi+a) & \text { if } \xi \geq-a+\delta \\ \log \delta+1+\frac{1}{2}|\xi+a|(\xi+a) & \text { if } \xi \leq-a+\delta\end{cases}
$$


Lemma 4.1. Assume $a>0$ and $0<\delta<1 / e$. Then

$$
\begin{aligned}
& \left|\Phi_{a, \delta}^{\prime}(\xi)\right| \leq|\log \delta|+2 a^{2}+2|\xi|^{2} \\
& \left(\Phi_{a, \delta}^{\prime}\left(\xi_{2}\right)-\Phi_{a, \delta}^{\prime}\left(\xi_{1}\right)\right)\left(\xi_{2}-\xi_{1}\right) \geq \frac{1}{4}\left|\xi_{2}-\xi_{1}\right|^{3} \\
& \Phi_{a, \delta}^{\prime}(\xi) \xi \geq \frac{1}{4}|\xi|^{3}-2-4 a^{3}
\end{aligned}
$$

for every $\xi, \xi_{1}, \xi_{2} \in \mathbb{R}$. Moreover

$$
\begin{aligned}
& \Phi_{a, \delta}^{\prime}(\xi) \xi \geq a|\log (\xi+a)|+\frac{1}{2}|\xi|^{3}-2-2 a^{3} \quad \text { if } \delta \leq \xi+a \leq 1, \\
& \Phi_{a, \delta}^{\prime}(\xi) \xi \geq a|\log \delta|+\frac{1}{4}|\xi|^{3}-2-4 a^{3} \quad \text { if } \xi+a \leq \delta, \\
& \Phi_{a, \delta}^{\prime}(\xi) \xi \geq c_{1}\left|\Phi_{a, \delta}^{\prime}(\xi)\right|-c_{2} \quad \text { for every } \xi \in \mathbb{R},
\end{aligned}
$$

where the constants $c_{1}>0$ and $c_{2}>0$ depend on a, but not on $\delta$.

Proof. Step 1: We prove (4.5). Using the inequality $0 \leq \log s \leq s^{2} / 2$ for $s \geq 1$, from (4.4) we obtain

$$
\left|\Phi_{a, \delta}^{\prime}(\xi)\right| \leq 1+|\xi+a|^{2} \quad \text { for } \xi+a \geq 1 .
$$

Since $|\log \delta| \geq 1$, this implies

$$
\left|\Phi_{a, \delta}^{\prime}(\xi)\right| \leq|\log \delta|+|\xi+a|^{2}
$$

On the other hand, if $\delta \leq \xi+a \leq 1$ we have $-|\log \delta| \leq \log (\xi+a)+1 \leq 1 \leq|\log \delta|$, so that (4.12) holds also in this case. Finally, (4.12) follows immediately from (4.4) if $\xi+a \leq \delta$. Hence, (4.5) is a consequence of (4.12) and Cauchy's Inequality.

Step 2: To show (4.6), note that, since $\Phi_{a, \delta}(\xi)-\frac{1}{6}|\xi+a|^{3}$ is convex, we have

$$
\left[\Phi_{a, \delta}^{\prime}\left(\xi_{2}\right)-\Phi_{a, \delta}^{\prime}\left(\xi_{1}\right)\right]\left(\xi_{2}-\xi_{1}\right) \geq \frac{1}{2}\left[\left|\xi_{2}+a\right|\left(\xi_{2}+a\right)-\left|\xi_{1}+a\right|\left(\xi_{1}+a\right)\right]\left(\xi_{2}-\xi_{1}\right) \geq \frac{1}{4}\left|\xi_{2}-\xi_{1}\right|^{3}
$$

for every $\xi_{1}, \xi_{2} \in \mathbb{R}$, where the last inequality follows from a straightforward calculation.

Step 3: To prove (4.7) we consider several cases.

Case 1: Assume first that $\xi+a \leq 0$. Then $\xi(\log \delta+1) \geq-a(\log \delta+1)=a|\log \delta|-a$ and $|\xi+a|(\xi+a) \xi=(\xi+a)^{2}|\xi| \geq|\xi|^{3}-2 a|\xi|^{2} \geq \frac{1}{2}|\xi|^{3}-5 a^{3}$, so that (4.4) implies

$$
\Phi_{a, \delta}^{\prime}(\xi) \xi \geq a|\log \delta|-a+\frac{1}{4}|\xi|^{3}-3 a^{3} \geq a|\log \delta|+\frac{1}{4}|\xi|^{3}-1-4 a^{3} .
$$

Case 2: Consider next the case $\xi+a>0$.

If $-a<\xi \leq 0$, then $(\xi+a)^{2} \xi \geq-a^{3} \geq|\xi|^{3}-2 a^{3}$, while if $\xi>0$, then $(\xi+a)^{2} \xi \geq|\xi|^{3}$. Therefore

$$
\xi+\frac{1}{2}(\xi+a)^{2} \xi \geq-a+\frac{1}{2}(\xi+a)^{2} \xi \geq-a+\frac{1}{2}|\xi|^{3}-a^{3} \geq \frac{1}{2}|\xi|^{3}-1-2 a^{3} .
$$

To estimate the logarithmic terms we consider three ranges of $\xi+a$. If $\xi+a \leq \delta$, then by (4.4) and (4.14),

$$
\begin{aligned}
\Phi_{a, \delta}^{\prime}(\xi) \xi & =\xi \log \delta+\xi+\frac{1}{2}(\xi+a)^{2} \xi \\
& \geq(\delta-a) \log \delta+\frac{1}{2}|\xi|^{3}-1-2 a^{3} \geq a|\log \delta|-\frac{1}{e}+\frac{1}{2}|\xi|^{3}-1-2 a^{3} \\
& \geq a|\log \delta|+\frac{1}{2}|\xi|^{3}-2-2 a^{3} .
\end{aligned}
$$

In the case $\delta<\xi+a \leq 1$ we have $(\xi+a) \log (\xi+a) \geq-1 / e$, hence $\xi \log (\xi+a) \geq$ $a|\log (\xi+a)|-1$. It follows from (4.4) and (4.14) that

$$
\Phi_{a, \delta}^{\prime}(\xi) \xi \geq a|\log (\xi+a)|-1+\xi+\frac{1}{2}(\xi+a)^{2} \xi \geq a|\log (\xi+a)|+\frac{1}{2}|\xi|^{3}-2-2 a^{3} .
$$

Finally, if $1<\xi+a$ and $\xi \leq 0$, then $0<\log (\xi+a) \leq \xi+a$, hence $\xi \log (\xi+a) \geq$ $\xi(\xi+a) \geq-a^{2}$, while if $1<\xi+a$ and $0<\xi$, then $\xi \log (\xi+a)>0$. In both cases, (4.4) and (4.14) give

$$
\Phi_{a, \delta}^{\prime}(\xi) \xi \geq-a^{2}+\xi+\frac{1}{2}(\xi+a)^{2} \xi \geq \frac{1}{2}|\xi|^{3}-2-3 a^{3} .
$$

Step 4: Note that (4.8) is exactly (4.16). Inequality (4.9) follows from (4.13) and (4.15). To prove (4.10), again we consider three ranges of $\xi+a$. In the case $\xi+a \leq \delta$ inequality 
(4.10) follows from (4.5) and (4.9). When $\delta<\xi+a \leq 1$ the same inequality is a consequence of (4.4) and (4.8). Finally, for $1<\xi+a$ inequality (4.10) can be obtained from (4.7) and (4.11).

We recall that $V:=W_{\text {per }_{0}}^{2,3}(I), Y:=L_{\operatorname{per}_{0}}^{2}(I)$, and $\|u\|_{V}:=\left\|u_{x x}\right\|_{L^{3}(I)}$. We introduce the operator $\mathcal{A}_{a, \delta}: V \rightarrow V^{\prime}$ defined by

$$
\left\langle\mathcal{A}_{a, \delta}(u), v\right\rangle_{V^{\prime}, V}:=\int_{I} \Phi_{a, \delta}^{\prime}\left(u_{x x}\right) v_{x x} d x
$$

for every $u, v \in V$. Note that by (4.5) and Hölder's and Minkowski's Inequalities, the operator $\mathcal{A}_{a, \delta}$ is well-defined and

$$
\left\|\mathcal{A}_{a, \delta}(u)\right\|_{V^{\prime}} \leq(2 \pi)^{2 / 3}\left(|\log \delta|+2 a^{2}\right)+2\|u\|_{V}^{2}
$$

for every $u \in V$. Moreover, by (4.7) we have

$$
\left\langle\mathcal{A}_{a, \delta}(u), u\right\rangle_{V^{\prime}, V} \geq \frac{1}{4}\|u\|_{V}^{3}-\left(4+8 a^{3}\right) \pi .
$$

Finally, (4.6) gives

$$
\left\langle\mathcal{A}_{a, \delta}\left(u^{2}\right)-\mathcal{A}_{a, \delta}\left(u^{1}\right), u^{2}-u^{1}\right\rangle_{V^{\prime}, V} \geq \frac{1}{4}\left\|u^{2}-u^{1}\right\|_{V}^{3}
$$

for every $u^{1}, u^{2} \in V$.

Therefore $\mathcal{A}_{a, \delta}$ is a bounded monotone operator. Moreover, by the continuity properties of Nemitski operators we deduce from (4.5) that $\mathcal{A}_{a, \delta}$ is continuous from $V$ to $V^{\prime}$.

Next we state the main theorem of this section. We recall that the operator $\mathcal{H}$ is defined in $(1.9)$.

Theorem 4.2. Let $a>0$ and let $u^{0} \in L_{\text {per }_{0}}^{2}(I)$. For every $0<\delta<1 /$ e there exists $a$ solution $u^{\delta}$ of the problem

$$
\left\{\begin{array}{l}
\dot{u}^{\delta}(t)+\mathcal{A}_{a, \delta}\left(u^{\delta}(t)\right)=\mathcal{H}\left(u^{\delta}(t)\right) \quad \text { for a.e. } t \in[0, T] \\
u^{\delta} \in L^{3}\left([0, T] ; W_{\operatorname{per}_{0}}^{2,3}(I)\right), \dot{u}^{\delta} \in L^{3 / 2}\left([0, T] ; W_{\text {per }_{0}}^{-2,3 / 2}(I)\right), \\
u^{\delta}(0)=u^{0}
\end{array}\right.
$$

Moreover, there exists a constant $c=c\left(a, T,\left\|u^{0}\right\|_{Y}\right)>0$ such that for every $0<\delta<1 / e$,

$$
\left\|u^{\delta}\right\|_{L^{3}([0, T] ; V)} \leq c, \quad\left\|\left(H\left(u_{x x}^{\delta}\right)\right)_{x}\right\|_{L^{3 / 2}\left([0, T] ; V^{\prime}\right)} \leq c .
$$

Proof. Step 1: We obtain apriori estimates for the solution of the auxiliary problem

$$
\left\{\begin{array}{l}
\dot{u}(t)+\mathcal{A}_{a, \delta}(u(t))=f(t) \quad \text { for a.e. } t \in[0, T] \\
u \in L^{3}([0, T] ; V), \dot{u} \in L^{3 / 2}\left([0, T] ; V^{\prime}\right) \\
u(0)=u^{0}
\end{array}\right.
$$

where $f \in L^{3 / 2}\left([0, T] ; V^{\prime}\right)$ and $u^{0} \in Y$. In order to guarantee the existence and uniqueness of the solution of problem (4.24), we use a slight extension of [5, Chapter 2, Theorem 1.2], where the estimates in (1.34) and (1.36) in this reference are replaced by (4.19) and (4.20), respectively. The proof is essentially the same.

Substep 1 a: Let $f^{1}, f^{2} \in L^{3 / 2}\left([0, T] ; V^{\prime}\right)$, and let $u^{1}, u^{2}$ be the corresponding solutions of (4.24) with the same initial value $u^{0}$. We claim that

$$
\left\|u^{2}-u^{1}\right\|_{L^{3}([0, T] ; V)}^{2} \leq 4\left\|f^{2}-f^{1}\right\|_{L^{3 / 2}\left([0, T] ; V^{\prime}\right)} .
$$

We multiply both equations by $u^{2}(t)-u^{1}(t)$ and subtract the first from the second to get

$$
\begin{aligned}
& \left\langle\dot{u}^{2}(t)-\dot{u}^{1}(t), u^{2}(t)-u^{1}(t)\right\rangle_{V^{\prime}, V}+\left\langle\mathcal{A}_{a, \delta}\left(u^{2}(t)\right)-\mathcal{A}_{a, \delta}\left(u^{1}(t)\right), u^{2}(t)-u^{1}(t)\right\rangle_{V^{\prime}, V} \\
& =\left\langle f^{2}(t)-f^{1}(t), u^{2}(t)-u^{1}(t)\right\rangle_{V^{\prime}, V}
\end{aligned}
$$


for a.e. $t \in[0, T]$. Since $t \mapsto \frac{1}{2}\left\|u^{2}(t)-u^{1}(t)\right\|_{Y}^{2}$ is absolutely continuous and its derivative equals $\left\langle\dot{u}^{2}(t)-\dot{u}^{1}(t), u^{2}(t)-u^{1}(t)\right\rangle_{V^{\prime}, V}$ for a.e. $t \in[0, T]$ (see, e.g., [10, Proposition 23.23]), integrating the previous equality from 0 to $T$ we deduce that

$$
\begin{aligned}
& \frac{1}{2}\left\|u^{2}(T)-u^{1}(T)\right\|_{Y}^{2}+\int_{0}^{T}\left\langle\mathcal{A}_{a, \delta}\left(u^{2}(t)\right)-\mathcal{A}_{a, \delta}\left(u^{1}(t)\right), u^{2}(t)-u^{1}(t)\right\rangle_{V^{\prime}, V} d t \\
& =\int_{0}^{T}\left\langle f^{2}(t)-f^{1}(t), u^{2}(t)-u^{1}(t)\right\rangle_{V^{\prime}, V} d t .
\end{aligned}
$$

By (4.21) we obtain

$$
\frac{1}{4}\left\|u^{2}-u^{1}\right\|_{L^{3}([0, T] ; V)}^{3} \leq\left\|f^{2}-f^{1}\right\|_{L^{3 / 2}\left([0, T] ; V^{\prime}\right)}\left\|u^{2}-u^{1}\right\|_{L^{3}([0, T] ; V)},
$$

and so (4.25) holds.

Substep 1 b: Let $f \in L^{3 / 2}\left([0, T] ; V^{\prime}\right)$, let $u^{0} \in Y$, and let $u$ be the corresponding solution of (4.24). We prove that

$$
\begin{aligned}
& \|u\|_{\mathcal{W}} \leq(2 \pi T)^{2 / 3}\left(|\log \delta|+2 a^{2}\right)+25\|f\|_{L^{3 / 2}\left([0, T] ; V^{\prime}\right)}+2\|f\|_{L^{3 / 2}\left([0, T] ; V^{\prime}\right)}^{1 / 2} \\
& +24\left\|u^{0}\right\|_{Y}^{4 / 3}+2\left\|u^{0}\right\|_{Y}^{2 / 3}+8(1+a) T^{1 / 3}+384(1+a)^{2} T^{2 / 3}
\end{aligned}
$$

where $\mathcal{W}$ is defined in $(2.22)$.

We multiply the equation by $u(t)$ and argue as in the previous substep. Using (4.20) we obtain

$$
\frac{1}{4}\|u\|_{L^{3}([0, T] ; V)}^{3} \leq\|f\|_{L^{3 / 2}\left([0, T] ; V^{\prime}\right)}\|u\|_{L^{3}([0, T] ; V)}+\left(4+8 a^{3}\right) \pi T+\frac{1}{2}\left\|u^{0}\right\|_{Y}^{2},
$$

which, together with Young's Inequality, gives

$$
\|u\|_{L^{3}([0, T] ; V)} \leq 2\|f\|_{L^{3 / 2}\left([0, T] ; V^{\prime}\right)}^{1 / 2}+8(1+a) T^{1 / 3}+2\left\|u^{0}\right\|_{Y}^{2 / 3} .
$$

Since $\dot{u}(t)=f(t)-\mathcal{A}_{a, \delta}(u(t))$ for a.e. $t \in[0, T]$, from (4.19) we get

$$
\|\dot{u}\|_{L^{3 / 2}\left([0, T] ; V^{\prime}\right)} \leq\|f\|_{L^{3 / 2}\left([0, T] ; V^{\prime}\right)}+(2 \pi T)^{2 / 3}\left(|\log \delta|+2 a^{2}\right)+2\|u\|_{L^{3}([0, T] ; V)}^{2},
$$

which, together with (4.28), yields

$$
\begin{aligned}
& \|\dot{u}\|_{L^{3 / 2}\left([0, T], V^{\prime}\right)} \leq(2 \pi T)^{2 / 3}\left(|\log \delta|+2 a^{2}\right)+25\|f\|_{L^{3 / 2}\left([0, T] ; V^{\prime}\right)} \\
& +384(1+a)^{2} T^{2 / 3}+24\left\|u^{0}\right\|_{Y}^{4 / 3} .
\end{aligned}
$$

Inequality (4.27) follows from (2.23), (4.28), and (4.29).

Step 2: Fix $u^{0} \in Y$. We prove existence of a solution of (4.22) using a fixed point argument. We begin by observing that given $u \in L^{3}([0, T] ; V)$, the function $t \mapsto\left(H\left(u(t)_{x x}\right)\right)_{x}$ belongs to $L^{3 / 2}\left([0, T] ; V^{\prime}\right)$. Therefore, in view of Step 1 there exists a unique solution $v$ of the problem

$$
\left\{\begin{array}{l}
\dot{v}(t)+\mathcal{A}_{a, \delta}(v(t))=\mathcal{H}(u(t)) \quad \text { for a.e. } t \in[0, T], \\
v \in \mathcal{W}, \quad v(0)=u^{0}
\end{array}\right.
$$

Let $\mathcal{T}_{\delta}: L^{3}([0, T] ; V) \rightarrow L^{3}([0, T] ; V)$ be the operator defined by $\mathcal{T}_{\delta}(u):=v$. In order to apply Schauder's Fixed Point Theorem we need to establish the following properties:

$\mathcal{T}_{\delta}$ is continuous;

$\mathcal{T}_{\delta}$ is compact;

$\mathcal{T}_{\delta}$ has an invariant ball.

To prove (4.31) we show that $\mathcal{T}$ is Hölder's continuous. Indeed, let $u^{1}, u^{2} \in L^{3}([0, T] ; V)$. Then by Hölder's Inequality,

$$
\left\|u_{x x}^{2}-u_{x x}^{1}\right\|_{L^{2}\left([0, T] ; L^{2}(I)\right)} \leq(2 \pi T)^{1 / 6}\left\|u^{2}-u^{1}\right\|_{L^{3}([0, T] ; V)},
$$


and so, by Proposition 2.4, we have that

$$
\left\|H\left(u_{x x}^{2}\right)-H\left(u_{x x}^{1}\right)\right\|_{L^{2}\left([0, T] ; L^{2}(I)\right)} \leq C_{2}(2 \pi T)^{1 / 6}\left\|u^{2}-u^{1}\right\|_{L^{3}([0, T] ; V)} .
$$

Therefore, there exists a constant $c=c(T)>0$ such that

$$
\left\|\left(H\left(u_{x x}^{2}\right)\right)_{x}-\left(H\left(u_{x x}^{1}\right)\right)_{x}\right\|_{L^{3 / 2}\left([0, T] ; W_{\text {per }_{0}}^{-1,3 / 2}(I)\right)} \leq c\left\|u^{2}-u^{1}\right\|_{L^{3}([0, T] ; V)},
$$

hence

$$
\left\|\left(H\left(u_{x x}^{2}\right)\right)_{x}-\left(H\left(u_{x x}^{1}\right)\right)_{x}\right\|_{L^{3 / 2}\left([0, T] ; V^{\prime}\right)} \leq c\left\|u^{2}-u^{1}\right\|_{L^{3}([0, T] ; V)},
$$

so that (4.25) yields

$$
\left\|\mathcal{T}_{\delta}\left(u^{2}\right)-\mathcal{T}_{\delta}\left(u^{1}\right)\right\|_{L^{3}([0, T] ; V)}^{2} \leq 4 c\left\|u^{2}-u^{1}\right\|_{L^{3}([0, T] ; V)},
$$

which establishes (4.31).

Let us prove (4.32). Let $\left\{u^{n}\right\}$ be a bounded sequence in $L^{3}([0, T] ; V)$ and, for every $n$, let

$$
v^{n}:=\mathcal{T}_{\delta}\left(u^{n}\right) \quad \text { and } \quad g^{n}:=\left(H\left(u_{x x}^{n}\right)\right)_{x} .
$$

By (4.34) the sequence $\left\{g^{n}\right\}$ is bounded in $L^{3 / 2}\left([0, T] ; W_{\text {per }_{0}}^{-1,3 / 2}(I)\right)$, so, in particular, it is also bounded in $L^{3 / 2}\left([0, T] ; V^{\prime}\right)$. Hence, by $(4.27)$ the sequence $\left\{v^{n}\right\}$ is bounded in $\mathcal{W}$, and so, passing to a subsequence, we may assume that $\left\{v^{n}\right\}$ converges weakly in $\mathcal{W}$. On the other hand, by Lemma 2.3, passing to a further subsequence, we may assume that $\left\{i^{*}\left(g^{n}\right)\right\}$ converges strongly in $\mathcal{W}^{\prime}$, where $i^{*}: L^{3 / 2}\left([0, T] ; W_{\text {per }_{0}}^{-1,3 / 2}(I)\right) \rightarrow \mathcal{W}^{\prime}$ is the embedding defined by (2.24). Arguing as in (4.26), we obtain

$\int_{0}^{T}\left\langle\mathcal{A}_{a, \delta}\left(v^{n}(t)\right)-\mathcal{A}_{a, \delta}\left(v^{m}(t)\right), v^{n}(t)-v^{m}(t)\right\rangle_{V^{\prime}, V} d t \leq \int_{0}^{T}\left\langle g^{n}(t)-g^{m}(t), v^{n}(t)-v^{m}(t)\right\rangle_{V^{\prime}, V} d t$ for every $n$ and $m$, and by (4.21) and (2.24) we have

$$
\left\|v^{n}-v^{m}\right\|_{L^{3}([0, T] ; V)}^{3} \leq 4\left\langle i^{*}\left(g^{n}\right)-i^{*}\left(g^{n}\right), v^{n}-v^{m}\right\rangle_{\mathcal{W}^{\prime}, \mathcal{W}} .
$$

Since the right-hand side of the previous inequality converges to zero as $n, m \rightarrow \infty$, it follows that $\left\{v^{n}\right\}$ is a Cauchy sequence in $L^{3}([0, T] ; V)$. This concludes the proof of (4.32).

To prove (4.33), we fix $R>0$. By (4.35), for every $u \in L^{3}([0, T] ; V)$ with $\|u\|_{L^{3}([0, T] ; V)} \leq$ $R$ we have

$$
\left\|\left(H\left(u_{x x}\right)\right)_{x}\right\|_{L^{3 / 2}\left([0, T] ; V^{\prime}\right)} \leq c\|u\|_{L^{3}([0, T] ; V)} \leq c R,
$$

Therefore, using (4.28) we get

$$
\left\|\mathcal{T}_{\delta}(u)\right\|_{L^{3}([0, T] ; V)} \leq 2(c R)^{1 / 2}+8(1+a) T^{1 / 3}+2\left\|u^{0}\right\|_{Y}^{2 / 3} .
$$

By taking $R=R\left(a, T,\left\|u^{0}\right\|_{Y}\right)$ sufficiently large we obtain that the right-hand side of the previous inequality is less than $R$. This concludes the proof of (4.33).

In view of (4.31), (4.32), and (4.33), by Schauder's Fixed Point Theorem the operator $\mathcal{T}_{\delta}$ has a fixed point, which is a solution of problem (4.22). Moreover, (4.23) follows from (4.36) and (4.37).

\section{An Auxiliary Problem}

Let $a>0$ and let $u^{0} \in L_{\text {per }_{0}}^{2}(I)$. For every $0<\delta<1 / e$ let $f^{\delta} \in L^{3 / 2}\left([0, T] ; W_{\text {per }_{0}}^{-2,3 / 2}(I)\right)$ and let $u^{\delta}$ be the solution of the problem

$$
\left\{\begin{array}{l}
\dot{u}^{\delta}(t)+\mathcal{A}_{a, \delta}\left(u^{\delta}(t)\right)=f^{\delta}(t) \quad \text { for a.e. } t \in[0, T] \\
u^{\delta} \in L^{3}\left([0, T] ; W_{\text {per }_{0}}^{2,3}(I)\right), \dot{u}^{\delta} \in L^{3 / 2}\left([0, T] ; W_{\text {per }_{0}}^{-2,3 / 2}(I)\right) \\
u^{\delta}(0)=u^{0}
\end{array}\right.
$$


Lemma 5.1. Let $a>0$ and let $u^{0} \in L_{\operatorname{per}_{0}}^{2}(I)$. Assume that $\left\{f^{\delta}\right\}_{0<\delta<1 / e}$ is bounded in $L^{3 / 2}\left([0, T] ; W_{\text {per }_{0}}^{-2,2}(I)\right)$, and for every $0<\delta<1 /$ e let $u^{\delta}$ be the solution of problem (5.1). Then there exists a constant $M$ such that

$$
\begin{aligned}
& \left\|u^{\delta}\right\|_{\left.L^{3}\left([0, T] ; W_{\text {per }_{0}}^{2,3}(I)\right)\right)} \leq M \\
& \int_{0}^{T}\left\langle\mathcal{A}_{a, \delta}\left(u^{\delta}(t)\right), u^{\delta}(t)\right\rangle_{V^{\prime}, V} d t \leq M
\end{aligned}
$$

for every $0<\delta<1 / e$.

Proof. Inequality (5.2) follows from (4.28). To prove the other inequality, for a.e. $t \in[0, T]$ we multiply the equation in (5.1) by $u_{\delta}(t)$, obtaining

$$
\left\langle\dot{u}^{\delta}(t), u^{\delta}(t)\right\rangle_{V^{\prime}, V}+\left\langle\mathcal{A}_{a, \delta}\left(u^{\delta}(t)\right), u^{\delta}(t)\right\rangle_{V^{\prime}, V}=\left\langle f^{\delta}(t), u^{\delta}(t)\right\rangle_{V^{\prime}, V} .
$$

Since $t \mapsto \frac{1}{2}\|u(t)\|_{L^{2}(I)}^{2}$ is absolutely continuous and its derivative equals $\langle\dot{u}(t), u(t)\rangle_{V^{\prime}, V}$ for a.e. $t \in[0, T]$ (see, e.g., [10, Proposition 23.23]), integrating the previous equality from 0 to $T$ we deduce that

$$
\begin{aligned}
& \frac{1}{2}\left\|u^{\delta}(T)\right\|_{L^{2}(I)}^{2}+\int_{0}^{T}\left\langle\mathcal{A}_{a, \delta}\left(u^{\delta}(t)\right), u^{\delta}(t)\right\rangle_{V^{\prime}, V} d t \\
& \quad \leq\left\|f^{\delta}\right\|_{L^{3 / 2}\left([0, T] ; V^{\prime}\right)}\left\|u^{\delta}\right\|_{L^{3}([0, T] ; V)}+\frac{1}{2}\left\|u^{0}\right\|_{L^{2}(I)}^{2} .
\end{aligned}
$$

By (5.2), this implies (5.3).

By (5.2) there exist a subsequence, still denoted $\left\{u^{\delta}\right\}$, and a function $\left.u \in L^{3}\left([0, T] ; W_{\text {per }_{0}}^{2,3}(I)\right)\right)$, such that

$$
\left.u^{\delta} \rightarrow u \quad \text { weakly in } L^{3}\left([0, T] ; W_{\text {per }_{0}}^{2,3}(I)\right)\right) .
$$

In the sequel (see Lemma 5.4) we will prove that, if $\left\{f^{\delta}\right\}_{0<\delta<1 / e}$ strongly converges to some function $f$ in $L^{3 / 2}\left([0, T] ; W_{\text {per }_{0}}^{-2,3 / 2}(I)\right)$, then $u$ is a weak solution of the limit problem, in the sense that

$$
\int_{0}^{T}\left(\langle\dot{v}(t), v(t)-u(t)\rangle_{V^{\prime}, V}+\mathcal{F}_{a}(v(t))\right) d t \geq \int_{0}^{T}\left(\mathcal{F}_{a}(u(t))+\langle f(t), v(t)-u(t)\rangle_{V^{\prime}, V}\right) d t
$$

for every $v \in \mathcal{W}$ with $v(0)=u^{0}$, where $\mathcal{F}_{a}$ is the functional defined in (1.8).

Lemma 5.2. Under the assumptions of Lemma 5.1, for every $0<\delta<1 /$ and a.e. $t \in[0, T]$ let

$$
\begin{aligned}
& E^{\delta}(t):=\left\{x \in I: u_{x x}^{\delta}(t, x)+a \leq \delta\right\}, \\
& F^{\delta}(t):=\left\{x \in I: \delta \leq u_{x x}^{\delta}(t, x)+a \leq 1\right\} .
\end{aligned}
$$

Then there exists a constant $M$ such that

$$
\begin{aligned}
& \int_{0}^{T} \mathcal{L}^{1}\left(E_{\delta}(t)\right) d t \leq M|\log \delta|^{-1} \\
& \int_{0}^{T}\left(\int_{F^{\delta}(t)}\left|\log \left(u^{\delta}(t)_{x x}+a\right)\right| d x\right) d t \leq M
\end{aligned}
$$

for every $0<\delta<1 / e$.

Proof. By (4.7) and (4.9), for a.e. $t \in[0, T]$ we have

$$
\left\langle\mathcal{A}_{a, \delta}\left(u^{\delta}(t)\right), u^{\delta}(t)\right\rangle_{V^{\prime}, V} \geq a|\log \delta| \mathcal{L}^{1}\left(E^{\delta}(t)\right)-4 \pi-8 \pi a^{3} .
$$

Integrating in time and using (5.3) we get (5.9).

On the other hand, by (4.7) and (4.8) for a.e. $t \in[0, T]$ we have

$$
\left\langle\mathcal{A}_{a, \delta}\left(u^{\delta}(t)\right), u^{\delta}(t)\right\rangle_{V^{\prime}, V} \geq a \int_{F_{\delta}(t)}\left|\log \left(u^{\delta}(t)_{x x}+a\right)\right| d x-4 \pi-8 \pi a^{3} .
$$


Integrating in time and using (5.3) we get (5.10).

For $\delta>0$ consider the functionals $\mathcal{F}_{a}, \mathcal{F}_{a, \delta}: W_{\text {per }_{0}}^{2,3}(I) \rightarrow \mathbb{R} \cup\{+\infty\}$ and $\mathcal{G}_{a}, \mathcal{G}_{a, \delta}$ : $W_{\text {per }_{0}}^{2,3}(I) \rightarrow \mathbb{R} \cup\{+\infty\}$ defined by

$$
\begin{aligned}
\mathcal{F}_{a}(u) & :=\int_{I} \Phi_{a}\left(u_{x x}\right) d x, & \mathcal{F}_{a, \delta}(u) & :=\int_{I} \Phi_{a, \delta}\left(u_{x x}\right) d x, \\
\mathcal{G}_{a}(u) & :=\int_{I} \Psi_{a}\left(u_{x x}\right) d x, & \mathcal{G}_{a, \delta}(u) & :=\int_{I} \Psi_{a, \delta}\left(u_{x x}\right) d x,
\end{aligned}
$$

where $\Phi_{a}, \Phi_{a, \delta}$, and $\Psi_{a}, \Psi_{a, \delta}$ are given in (1.6), (4.3), (1.6), and (4.2), respectively.

Lemma 5.3. Under the assumptions of Lemma 5.1, for every $0<\delta<1 /$ e we have

$$
\int_{0}^{T}\left(\left\langle\dot{v}(t), v(t)-u^{\delta}(t)\right\rangle_{V^{\prime}, V}+\mathcal{F}_{a, \delta}(v(t))-\mathcal{F}_{a, \delta}\left(u^{\delta}(t)\right)\right) d t \geq \int_{0}^{T}\left\langle f^{\delta}(t), v(t)-u^{\delta}(t)\right\rangle_{V^{\prime}, V} d t
$$

for every $v \in \mathcal{W}$ with $v(0)=u^{0}$.

Proof. Fix $0<\delta<1 / e$ and $v \in \mathcal{W}$ with $v(0)=u^{0}$. For a.e. $t \in[0, T]$ we multiply equation (5.1) by $v(t)-u^{\delta}(t)$. Adding $\left\langle\dot{v}(t), v(t)-u^{\delta}(t)\right\rangle_{V^{\prime}, V}$ to both sides we get

$$
\begin{aligned}
\langle\dot{v}(t), & \left.v(t)-u^{\delta}(t)\right\rangle_{V^{\prime}, V}+\left\langle\mathcal{A}_{a, \delta}\left(u^{\delta}(t)\right), v(t)-u^{\delta}(t)\right\rangle_{V^{\prime}, V} \\
& =\left\langle\dot{v}(t)-\dot{u}^{\delta}(t), v(t)-u^{\delta}(t)\right\rangle_{V^{\prime}, V}+\left\langle f^{\delta}(t), v(t)-u^{\delta}(t)\right\rangle_{V^{\prime}, V} .
\end{aligned}
$$

Since $\mathcal{A}_{a, \delta}=\partial \mathcal{F}_{a, \delta}$ and $\mathcal{F}_{a, \delta}$ is convex, we obtain

$$
\begin{aligned}
& \left\langle\dot{v}(t), v(t)-u^{\delta}(t)\right\rangle_{V^{\prime}, V}+\mathcal{F}_{a, \delta}(v(t))-\mathcal{F}_{a, \delta}\left(u^{\delta}(t)\right) \\
& \quad \geq \frac{1}{2} \frac{d}{d t}\left\|v(t)-u^{\delta}(t)\right\|_{L^{2}(I)}^{2}+\left\langle f^{\delta}(t), v(t)-u^{\delta}(t)\right\rangle_{V^{\prime}, V},
\end{aligned}
$$

where we have also used [10, Proposition 23.23]. Integrating with respect to $t$ we obtain (5.13).

Lemma 5.4. Under the hypotheses of Lemma 5.1, let $u$ be the function defined in (5.5). Assume that $\left\{f^{\delta}\right\}_{0<\delta<1 / e}$ strongly converges to some function $f$ in $L^{3 / 2}\left([0, T] ; W_{\text {per }_{0}}^{-2,3 / 2}(I)\right)$. Then $u$ satisfies (5.6).

Proof. By (5.5) we have

$$
\begin{aligned}
\int_{0}^{T}\left\langle\dot{v}(t), v(t)-u^{\delta}(t)\right\rangle_{V^{\prime}, V} d t & \rightarrow \int_{0}^{T}\langle\dot{v}(t), v(t)-u(t)\rangle_{V^{\prime}, V} d t \\
\int_{0}^{T}\left\langle f^{\delta}(t), v(t)-u^{\delta}(t)\right\rangle_{V^{\prime}, V} d t & \rightarrow \int_{0}^{T}\langle f(t), v(t)-u(t)\rangle_{V^{\prime}, V} d t
\end{aligned}
$$

On the other hand,

$$
\int_{0}^{T} \mathcal{F}_{a}(v(t)) d t \geq \int_{0}^{T} \mathcal{F}_{a, \delta}(v(t)) d t
$$

because $\mathcal{F}_{a} \geq \mathcal{F}_{a, \delta}$.

Finally, in order to study the term $\mathcal{F}_{a, \delta}\left(u^{\delta}(t)\right)$ we fix $0<\eta<1 / e$ and we use the inequality

$$
\mathcal{G}_{a, \delta}\left(u^{\delta}(t)\right) \geq \mathcal{G}_{a, \eta}\left(u^{\delta}(t)\right)
$$

for every $0<\delta<\eta$. Since the functional

$$
w \mapsto \int_{0}^{T} \mathcal{G}_{a, \delta}(w) d t
$$


is convex and continuous for the strong topology of $L^{3}([0, T] ; V)$, it is also lower semicontinuous for the weak topology of $L^{3}([0, T] ; V)$. By (5.5) and (5.17) this implies

$$
\liminf _{\delta \rightarrow 0} \int_{0}^{T} \mathcal{G}_{a, \delta}\left(u^{\delta}(t)\right) d t \geq \liminf _{\delta \rightarrow 0} \int_{0}^{T} \mathcal{G}_{a, \eta}\left(u^{\delta}(t)\right) d t \geq \int_{0}^{T} \mathcal{G}_{a, \eta}(u(t)) d t .
$$

Taking the limit as $\eta \rightarrow 0$ and using the Monotone Convergence Theorem, we get

$$
\liminf _{\delta \rightarrow 0} \int_{0}^{T} \mathcal{G}_{a, \delta}\left(u^{\delta}(t)\right) d t \geq \int_{0}^{T} \mathcal{G}_{a}(u(t)) d t .
$$

Adding the cube of the norm in $\left.L^{3}([0, T]) ; V\right)$ we obtain

$$
\liminf _{\delta \rightarrow 0} \int_{0}^{T} \mathcal{F}_{a, \delta}\left(u^{\delta}(t)\right) d t \geq \int_{0}^{T} \mathcal{F}_{a}(u(t)) d t .
$$

Inequality (5.6) follows now from $(5.13),(5.14),(5.15),(5.16)$, and (5.19).

From now on we assume that there exists $w^{0} \in \mathcal{W}$ such that $w^{0}(0)=u^{0}$ and

$$
\int_{0}^{T} \mathcal{F}_{a}\left(w^{0}(t)\right) d t<+\infty
$$

Then (5.6) implies that

$$
\int_{0}^{T} \mathcal{F}_{a}(u(t)) d t<+\infty
$$

Lemma 5.5. Let $u^{0} \in L_{\text {per }_{0}}^{2}(I)$ and let $w \in L^{3}\left([0, T] ; W_{\text {per }_{0}}^{2,3}(I)\right)$ be such that for a.e. $t \in[0, T]$ the function $w_{x x}(t)+a$ is nonnegative. Then there exists a sequence $\left\{w^{n}\right\}$ in $\mathcal{W}$ such that

$$
w^{n}(0)=u^{0} \quad \text { for every } n,
$$

for every $t \in[0, T]$ and every $n \in \mathbb{N}$ the function $w_{x x}^{n}(t)+a$ is nonnegative,

$$
\begin{aligned}
& \limsup _{n \rightarrow \infty} \int_{0}^{T}\left\langle\dot{w}^{n}(t), w^{n}(t)-w(t)\right\rangle_{V^{\prime}, V} d t \leq 0, \\
& w^{n} \rightarrow w \text { strongly in } L^{3}\left([0, T] ; W_{\text {per }_{0}}^{2,3}(I)\right), \\
& \lim _{n \rightarrow \infty} \int_{0}^{T} \mathcal{F}_{a}\left(w^{n}(t)\right) d t=\int_{0}^{T} \mathcal{F}_{a}(w(t)) d t .
\end{aligned}
$$

Proof. Let $w^{0} \in \mathcal{W}$ be the function in (5.20). Since $w^{0} \in L^{3}([0, T] ; V) \cap C^{0}([0, T] ; H)$, there exists a sequence $\delta_{n} \searrow 0$ such that

$$
\delta_{n}\left\|w^{0}\left(\delta_{n}\right)\right\|_{V}^{3} \rightarrow 0
$$

Define

$$
w^{n}(t):= \begin{cases}w^{0}(t) & \text { for } t \in\left[0, \delta_{n}\right], \\ \frac{1}{\delta_{n}} \int_{\delta_{n}}^{t} e^{-(t-s) / \delta_{n}} w(s) d s+e^{-\left(t-\delta_{n}\right) / \delta_{n}} w^{0}\left(\delta_{n}\right) & \text { for } t \in\left[\delta_{n}, T\right] .\end{cases}
$$

Then $w^{n} \in \mathcal{W}$ and (5.22) holds. Since $w_{x x}(t)+a \geq 0$ for a.e. $t \in[0, T]$ by hypothesis, and $w_{x x}^{0}(t)+a \geq 0$ for every $t \in[0, T]$ by (5.20) (recall that $w^{0}$ is continuous with values in $\left.L_{\text {per }_{0}}^{2}(I)\right)$, we have that (5.23) is satisfied. Moreover, we have

$$
\delta_{n} \dot{w}^{n}(t)=w(t)-w^{n}(t) \quad \text { for a.e. } t \in\left[\delta_{n}, T\right] .
$$

Hence,

$$
\delta_{n}\left\langle\dot{w}^{n}(t), w^{n}(t)-w(t)\right\rangle_{V^{\prime}, V}=-\left\langle w^{n}(t)-w(t), w^{n}(t)-w(t)\right\rangle_{V^{\prime}, V} \leq 0
$$


for a.e. $t \in\left[\delta_{n}, T\right]$. Therefore,

$$
\int_{0}^{T}\left\langle\dot{w}^{n}(t), w^{n}(t)-w(t)\right\rangle_{V^{\prime}, V} d t \leq \int_{0}^{\delta_{n}}\left\langle\dot{w}^{0}(t), w^{0}(t)-w(t)\right\rangle_{V^{\prime}, V} d t .
$$

Since $w^{0} \in \mathcal{W}$ and $w \in L^{3}\left([0, T] ; W_{\text {per }_{0}}^{2,3}(I)\right)$, inequality (5.24) follows.

Let us prove (5.25). We observe that

$$
w^{n}(t)-w(t)=a^{n}(t)+b^{n}(t),
$$

with

$$
\begin{aligned}
a^{n}(t) & :=\frac{1}{\delta_{n}} \int_{\delta_{n}}^{t} e^{-(t-s) / \delta_{n}}(w(s)-w(t)) d s, \\
b^{n}(t) & :=e^{-\left(t-\delta_{n}\right) / \delta_{n}}\left(w^{0}\left(\delta_{n}\right)-w(t)\right),
\end{aligned}
$$

for every $t \in\left[\delta_{n}, T\right]$. From standard properties of convolutions, we deduce that

$$
\int_{\delta_{n}}^{T}\left\|a^{n}(t)\right\|_{V}^{3} d t \rightarrow 0
$$

while

$$
\int_{\delta_{n}}^{T}\left\|b^{n}(t)\right\|_{V}^{3} d t \rightarrow 0
$$

by (5.27) and by the Dominated Convergence Theorem. Since $w^{n}(t)-w(t)=w^{0}(t)-w(t)$ for every $t \in\left[0, \delta_{n}\right]$, we have also

$$
\int_{0}^{\delta_{n}}\left\|w^{n}(t)-w(t)\right\|_{V}^{3} d t \rightarrow 0
$$

Property (5.25) follows from (5.29)-(5.32). Equality (5.26) is a consequence of (5.25), in view of $(5.23)$.

Theorem 5.6. Let $a>0$, let $f \in L^{3 / 2}\left([0, T] ; W_{\text {per }_{0}}^{-2,3}(I)\right)$, and let $u^{0} \in L_{\text {per }_{0}}^{2}(I)$. Assume that (5.20) is satisfied. Then there exists a unique solution of $(5.6)$ in $L^{3}\left([0, T] ; W_{\text {per }_{0}}^{2,3}(I)\right)$.

Proof. The existence follows from (5.5) and Lemma 5.4 with $f_{\delta}:=f$ for all $\delta>0$. To prove uniqueness, let $u^{1}$ and $u^{2} \in L^{3}\left([0, T] ; W_{\text {per }_{0}}^{2,3}(I)\right)$ be solutions of $(5.6)$, and let $w:=$ $\frac{1}{2}\left(u^{1}+u^{2}\right)$. By $(5.20)$ we have

$$
\int_{0}^{T} \mathcal{F}_{a}\left(u^{1}(t)\right) d t<+\infty \text { and } \int_{0}^{T} \mathcal{F}_{a}\left(u^{2}(t)\right) d t<+\infty
$$

so that for a.e. $t \in[0, T]$ the functions $u_{x x}^{1}(t)+a$ and $u_{x x}^{2}(t)+a$ are nonnegative. Let $\left\{w^{n}\right\}$ be the sequence given by Lemma 5.5. We write (5.6) for $u^{1}$ and $u^{2}$ with $w^{n}$ in place of $v$. Adding the resulting inequalities, we obtain

$$
\begin{aligned}
\int_{0}^{T} & \left(\left\langle\dot{w}^{n}(t), w^{n}(t)-w(t)\right\rangle_{V^{\prime}, V}+\mathcal{F}_{a}\left(w^{n}(t)\right)\right) d t \\
& \geq \int_{0}^{T}\left(\frac{1}{2} \mathcal{F}_{a}\left(u^{1}(t)\right)+\frac{1}{2} \mathcal{F}_{a}\left(u^{2}(t)\right)+\left\langle f(t), w^{n}(t)-w(t)\right\rangle_{V^{\prime}, V}\right) d t
\end{aligned}
$$

Passing to the limit and using (5.24), (5.25), and (5.26) we obtain

$$
\int_{0}^{T} \mathcal{F}_{a}(w(t)) d t \geq \frac{1}{2} \int_{0}^{T} \mathcal{F}_{a}\left(u^{1}(t)\right) d t+\frac{1}{2} \int_{0}^{T} \mathcal{F}_{a}\left(u^{2}(t)\right) d t .
$$

Since $\mathcal{F}_{a}$ is strictly convex in $L^{3}\left([0, T] ; W_{\text {per }_{0}}^{2,3}(I)\right)$ (see $(1.2)$ and $(2.20)$ ), we conclude that $u^{1}=u^{2}$. 
Remark 5.7. Under the assumptions of this theorem, it follows that if $u^{\delta}$ is the solution of problem $(5.1), 0<\delta<1 / e$, and if $\left\{f^{\delta}\right\}_{0<\delta<1 / e}$ strongly converges to some function $f$ in $L^{3 / 2}\left([0, T] ; W_{\text {per }_{0}}^{-2,3 / 2}(I)\right)$, then the entire sequence $\left\{u^{\delta}\right\}_{0<\delta<1 / e}$ weakly converges in $L^{3}\left([0, T] ; W_{\text {per }_{0}}^{2,3}(I)\right)$ to the unique solution of $(5.6)$.

Proposition 5.8. Let $a>0$ and let $u^{0} \in L_{\operatorname{per}_{0}}^{2}(I)$. Assume that $\left\{f^{\delta}\right\}_{0<\delta<1 / e}$ strongly

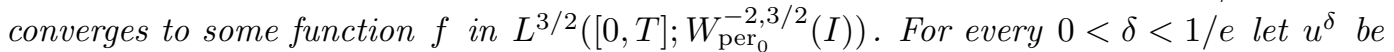
the solution of problem (5.1) and let $u$ be the solution of (5.6). Then

$$
\begin{gathered}
u^{\delta} \rightarrow u \quad \text { strongly in } L^{3}\left([0, T] ; W_{\text {per }_{0}}^{2,3}(I)\right), \\
\lim _{\delta \rightarrow 0} \int_{0}^{T} \mathcal{G}_{a, \delta}\left(u^{\delta}(t)\right) d t=\int_{0}^{T} \mathcal{G}_{a}(u(t)) d t .
\end{gathered}
$$

Proof. We prove that

$$
\lim _{\delta \rightarrow 0} \int_{0}^{T} \mathcal{F}_{a, \delta}\left(u^{\delta}(t)\right) d t=\int_{0}^{T} \mathcal{F}_{a}(u(t)) d t .
$$

In view of (5.19) it is enough to show that

$$
\limsup _{\delta \rightarrow 0} \int_{0}^{T} \mathcal{F}_{a, \delta}\left(u^{\delta}(t)\right) d t \leq \int_{0}^{T} \mathcal{F}_{a}(u(t)) d t .
$$

Let $\left\{w^{n}\right\}$ be the sequence given by Lemma 5.5 with $w=u$. By (5.13) we have

$\int_{0}^{T}\left(\left\langle\dot{w}^{n}(t), w^{n}(t)-u^{\delta}(t)\right\rangle_{V^{\prime}, V}+\mathcal{F}_{a, \delta}\left(w^{n}(t)\right)\right) d t \geq \int_{0}^{T}\left(\mathcal{F}_{a, \delta}\left(u^{\delta}(t)\right)+\left\langle f^{\delta}(t), w^{n}(t)-u^{\delta}(t)\right\rangle_{V^{\prime}, V}\right) d t$ for every $0<\delta<1 / e$ and every $n$. Taking the limit as $\delta \rightarrow 0$ and using the fact that $\mathcal{F}_{a} \geq \mathcal{F}_{a, \delta}$, we obtain

$$
\begin{aligned}
& \int_{0}^{T}\left(\left\langle\dot{w}^{n}(t), w^{n}(t)-u(t)\right\rangle_{V^{\prime}, V}+\mathcal{F}_{a}\left(w^{n}(t)\right)\right) d t \\
& \quad \geq \limsup _{\delta \rightarrow 0} \int_{0}^{T} \mathcal{F}_{a, \delta}\left(u^{\delta}(t)\right) d t+\int_{0}^{T}\left\langle f(t), w^{n}(t)-u(t)\right\rangle_{V^{\prime}, V} d t .
\end{aligned}
$$

Taking the limit as $n \rightarrow \infty$ and using (5.24), (5.23), and (5.25) we obtain (5.36), which gives (5.35). Hence, (5.35) holds, or, equivalently,

$\lim _{\delta \rightarrow 0}\left(\int_{0}^{T} \mathcal{G}_{a, \delta}\left(u^{\delta}(t)\right) d t+\frac{1}{6}\left\|u^{\delta}+a\right\|_{L^{3}\left([0, T] ; W_{\text {per }_{0}}^{2,3}(I)\right)}^{3}\right)=\int_{0}^{T} \mathcal{G}_{a}(u(t)) d t+\frac{1}{6}\|u+a\|_{L^{3}\left([0, T] ; W_{\text {per }_{0}}^{2,3}(I)\right)}^{3}$.

In view of (5.18) and of the weak lower semicontinuity of the norm, we obtain (5.34) and the convergence of $\left\|u^{\delta}+a\right\|_{L^{3}\left([0, T] ; W_{\left.\text {per }_{0}(I)\right)}^{2,3}\right.}$ to $\|u+a\|_{L^{3}\left([0, T] ; W_{\operatorname{per}_{0}}^{2,3}(I)\right)}$. By the uniform convexity of the norm of $L^{3}\left([0, T] ; W_{\text {per }_{0}}^{2,3}(I)\right)$, we deduce $(5.33)$.

Proposition 5.9. Under the assumptions of Theorem 5.6, the solution $u$ of problem (5.6) satisfies

$$
\log \left(u_{x x}+a\right) \in L^{1}\left([0, T] ; L^{1}(I)\right) .
$$

In particular, $u_{x x}+a>0$ a.e. on $[0, T] \times I$.

Proof. For every $0<\delta<1 / e$ we define

$$
\tilde{E}^{\delta}:=\left\{(t, x) \in[0, T] \times I: u_{x x}^{\delta}(t, x)+a \leq \delta\right\} .
$$

By Fubini's Theorem and by Lemma 5.2,

$$
\int_{[0, T] \times I} 1_{\tilde{E}^{\delta}}(t, x) d x d t=\int_{0}^{T} \mathcal{L}^{1}\left(E_{\delta}(t)\right) d t \rightarrow 0,
$$


and so there exists a subsequence (not relabeled) such that $1_{\tilde{E}^{\delta}} \rightarrow 0$ pointwise a.e. on $[0, T] \times I$. Since $u_{x x}^{\delta} \rightarrow u_{x x}$ strongly in $L^{3}\left([0, T] ; L_{\text {per }_{0}}^{3}(I)\right)$, passing to a further subsequence (still not relabeled) we obtain that $u_{x x}^{\delta} \rightarrow u_{x x}$ pointwise a.e. on $[0, T] \times I$.

Define

$G^{\delta}:=\left\{(t, x) \in[0, T] \times I: u_{x x}^{\delta}(t, x)+a<1\right\} \quad$ and $\quad G:=\left\{(t, x) \in[0, T] \times I: u_{x x}(t, x)+a<1\right\}$.

Then, for a.e. $(t, x) \in[0, T] \times I$,

$$
1_{G}(t, x) \leq \liminf _{\delta \rightarrow 0} 1_{G^{\delta}}(t, x) .
$$

Since $1_{G^{\delta}}(t, x) \leq 1_{\tilde{E}^{\delta}}(t, x)+1_{F^{\delta}(t)}(x)$, we conclude that

$$
1_{G}(t, x) \leq \lim _{\delta \rightarrow 0} 1_{\tilde{E}^{\delta}}(t, x)+\liminf _{\delta \rightarrow 0} 1_{F^{\delta}(t)}(x)=\liminf _{\delta \rightarrow 0} 1_{F^{\delta}(t)}(x),
$$

which gives

$$
1_{G}(t, x)\left|\log \left(u_{x x}(t, x)+a\right)\right| \leq \liminf _{\delta \rightarrow 0} 1_{F^{\delta}(t)}(x)\left|\log \left(u_{x x}^{\delta}(t, x)+a\right)\right| .
$$

By Fatou Lemma and Fubini's Theorem it follows from (5.10) that

$$
\begin{aligned}
\int_{G}\left|\log \left(u_{x x}(t, x)+a\right)\right| d x d t & \leq \int_{[0, T] \times I} \liminf _{\delta \rightarrow 0} 1_{F^{\delta}(t)}(x)\left|\log \left(u_{x x}^{\delta}(t, x)+a\right)\right| d x d t \\
& \leq \liminf _{\delta \rightarrow 0} \int_{[0, T] \times I} 1_{F^{\delta}(t)}(x)\left|\log \left(u_{x x}^{\delta}(t, x)+a\right)\right| d x d t \leq M .
\end{aligned}
$$

Using the fact that $u_{x x} \in L^{3}\left([0, T] ; L_{\text {per }_{0}}^{3}(I)\right)$, we deduce that $\left|\log \left(u_{x x}+a\right)\right|$ is integrable on the set $\left\{(t, x) \in[0, T] \times I: u_{x x}(t, x)+a \geq 1\right\}$, which, together with the previous inequality, gives the result.

Proposition 5.10. Under the assumptions of Theorem 5.6, the solution $u$ of problem (5.6) satisfies

$$
\int_{0}^{T}\left(\langle\dot{w}(t), w(t)-u(t)\rangle_{V^{\prime}, V}+\left\langle\mathcal{A}_{a}(u(t)), w(t)-u(t)\right\rangle_{2, \infty}\right) d t \geq \int_{0}^{T}\langle f(t), w(t)-u(t)\rangle_{V^{\prime}, V} d t
$$

for every $w \in \mathcal{W}$, with $w(0)=u^{0}$, such that

$$
\begin{aligned}
& w-u \in L^{\infty}\left([0, T] ; W^{2, \infty}(I)\right), \\
& \log \left(w_{x x}+a\right) \in L^{1}\left([0, T] ; L^{1}(I)\right) .
\end{aligned}
$$

Proof. Consider $w$ as in the statement of this proposition and fix $\varepsilon>0$. In view of Proposition 5.9, we can find a sequence $\left\{u^{n}\right\}$ converging to $u$ and satisfying all conditions of Lemma 5.5, with $w$ replaced by $u$. Define $v^{n}:=u^{n}+\varepsilon\left(w-u^{n}\right)$. Then we have

$$
\begin{aligned}
& \left\langle\dot{v}^{n}(t), v^{n}(t)-u(t)\right\rangle_{V^{\prime}, V}=(1-\varepsilon)\left\langle\dot{u}^{n}(t), u^{n}(t)-u(t)\right\rangle_{V^{\prime}, V}+\varepsilon(1-\varepsilon)\left\langle\dot{u}^{n}(t), w(t)-u^{n}(t)\right\rangle_{V^{\prime}, V} \\
& +\varepsilon\left\langle\dot{w}(t), u^{n}(t)-u(t)\right\rangle_{V^{\prime}, V}+\varepsilon^{2}\left\langle\dot{w}(t), w(t)-u^{n}(t)\right\rangle_{V^{\prime}, V} .
\end{aligned}
$$

For the second term, we add and subtract $\dot{w}(t)$ and we obtain

$$
\left\langle\dot{u}^{n}(t), w(t)-u^{n}(t)\right\rangle_{V^{\prime}, V}=-\frac{1}{2} \frac{d}{d t}\left\|w(t)-u^{n}(t)\right\|_{L^{2}(I)}^{2}+\left\langle\dot{w}(t), w(t)-u^{n}(t)\right\rangle_{V^{\prime}, V} .
$$

Upon integration and using the fact that the first term on the right-hand side of (5.41) is asymptotically nonpositive by (5.24), we get

$$
\int_{0}^{T}\left\langle\dot{v}^{n}(t), v^{n}(t)-u(t)\right\rangle_{V^{\prime}, V} d t \leq \varepsilon \int_{0}^{T}\langle\dot{w}(t), w(t)-u(t)\rangle_{V^{\prime}, V} d t+\delta_{n},
$$


where $\delta_{n} \searrow 0$. Setting $v=v^{n}$ in (5.6), we find

$$
\begin{aligned}
& \varepsilon \int_{0}^{T}\langle\dot{w}(t), w(t)-u(t)\rangle_{V^{\prime}, V} d t+\delta_{n}+\int_{0}^{T}\left(\mathcal{F}_{a}\left(v^{n}(t)\right)-\mathcal{F}_{a}(u(t))\right) d t \\
& \geq \int_{0}^{T}\left\langle f(t), v^{n}(t)-u(t)\right\rangle_{V^{\prime}, V} d t
\end{aligned}
$$

By (5.25), $\int_{0}^{T}\left\langle f(t), v^{n}(t)-u(t)\right\rangle_{V^{\prime}, V} d t \rightarrow \varepsilon \int_{0}^{T}\langle f(t), w(t)-u(t)\rangle_{V^{\prime}, V} d t$. Moreover, since for a.e. $t \in[0, T]$ the function $v_{x x}^{n}(t)+a$ is nonnegative by (5.23) and (5.40), reasoning as in the end of the proof of Lemma 5.5, we have that $\int_{0}^{T} \mathcal{F}_{a}\left(v^{n}(t)\right) d t \rightarrow \int_{0}^{T} \mathcal{F}_{a}(u(t)+\varepsilon(w(t)-$ $u(t))) d t$. Hence, passing to the limit as $n \rightarrow \infty$ and dividing by $\varepsilon$ we obtain

$$
\begin{aligned}
& \int_{0}^{T}\langle\dot{w}(t), w(t)-u(t)\rangle_{V^{\prime}, V} d t+\frac{1}{\varepsilon} \int_{0}^{T}\left(\mathcal{F}_{a}(u(t)+\varepsilon(w(t)-u(t)))-\mathcal{F}_{a}(u(t))\right) d t \\
& \geq \int_{0}^{T}\langle f(t), w(t)-u(t)\rangle_{V^{\prime}, V} d t
\end{aligned}
$$

By the Mean Value Theorem,

$$
\begin{aligned}
& \frac{\mathcal{F}_{a}(u(t)+\varepsilon(w(t)-u(t)))-\mathcal{F}_{a}(u(t))}{\varepsilon} \\
& =\int_{I} \Phi_{a}^{\prime}\left(u_{x x}(t)+\theta_{\varepsilon}(t, x) \varepsilon\left(w_{x x}(t)-u_{x x}(t)\right)\right)\left(w_{x x}(t)-u_{x x}(t)\right) d x,
\end{aligned}
$$

where $\theta_{\varepsilon}(t, x) \in(0,1)$. By (1.4), and using the fact that

$$
|\log (\theta x+(1-\theta) y)| \leq \begin{cases}|\log x|+|\log y| & \text { if } 0<\theta x+(1-\theta) y<1 \\ |x|+|y| & \text { otherwise, }\end{cases}
$$

in view of $(5.37),(5.39)$, and (5.40), we can apply the Dominated Convergence Theorem as $\varepsilon \rightarrow 0$ to obtain $(5.38)$.

\section{Proof of the Main Theorems}

In this section we prove Theorems 1.1 and 1.2. Let $a>0$ and let $u^{0} \in L_{\text {per }_{0}}^{2}(I)$. For every $0<\delta<1 / e$ let $u^{\delta}$ be the solution to problem (4.22) (see Theorem 4.2). Define

$$
f^{\delta}(t, x):=-\left(H\left(u_{x x}^{\delta}(t)\right)\right)_{x} .
$$

By (4.23), we apply Lemma 5.1 to obtain that (5.2) and (5.3) are satisfied. Using (4.10) and (5.3) we deduce that

$$
\left\|\mathcal{A}_{a, \delta}\left(u^{\delta}(t)\right)\right\|_{L^{1}\left([0, T] ;\left(W_{\text {per }_{0}}^{2, \infty}(I)\right)^{\prime}\right)} \leq M
$$

In turn, by $(4.22),(5.2),(6.1)$, and Proposition 2.4 we conclude that

$$
\left\|\dot{u}^{\delta}\right\|_{L^{1}\left([0, T] ;\left(W_{\text {per }_{0}}^{2, \infty}(I)\right)^{\prime}\right)} \leq M \text {. }
$$

By (5.2) there exist a subsequence (not relabeled) and a function $\left.u \in L^{3}\left([0, T] ; W_{\text {per }_{0}}^{2,3}(I)\right)\right)$, such that

$$
\left.u^{\delta} \rightarrow u \quad \text { weakly in } L^{3}\left([0, T] ; W_{\text {per }_{0}}^{2,3}(I)\right)\right) .
$$

In view of (6.2) we can now apply Theorem 2.1 with $B_{0}=W_{\text {per }_{0}}^{2,3}(I), B=W_{\text {per }_{0}}^{1,3}(I)$, $B_{1}=\left(W_{\text {per }_{0}}^{2, \infty}(I)\right)^{\prime}$, and $p=3$, obtaining that

$$
\left.u^{\delta} \rightarrow u \quad \text { strongly in } L^{3}\left([0, T] ; W_{\text {per }_{0}}^{1,3}(I)\right)\right),
$$

hence

$$
\left.u_{x x}^{\delta} \rightarrow u_{x x} \quad \text { strongly in } L^{3}\left([0, T] ; W_{\operatorname{per}_{0}}^{-1,3}(I)\right)\right)
$$


Since $H$ is a continuous linear operator from $\left.W_{\text {per }_{0}}^{-1,3}(I)\right)$ into itself (see Remark 2.6), we deduce that $\left(H\left(u_{x x}^{\delta}\right)\right)_{x} \rightarrow\left(H\left(u_{x x}\right)\right)_{x}$ strongly in $\left.L^{3}\left([0, T] ; W_{\text {per }_{0}}^{-2,3}(I)\right)\right)$, and so,

$$
\left.\left(H\left(u_{x x}^{\delta}\right)\right)_{x} \rightarrow\left(H\left(u_{x x}\right)\right)_{x} \quad \text { strongly in } L^{3 / 2}\left([0, T] ; W_{\operatorname{per}_{0}}^{-2,3 / 2}(I)\right)\right) .
$$

Hence, we can apply Lemma 5.4 to establish that $u$ satisfies (1.7) for every $w \in \mathcal{W}$ with $w(0)=u^{0}$. This concludes the proof of Theorem 1.1.

By Proposition 5.9 we have $\log \left(u_{x x}+a\right) \in L^{1}\left([0, T] ; L^{1}(I)\right)$ and by Proposition 5.10 we obtain

$\int_{0}^{T}\left(\langle\dot{w}(t), w(t)-u(t)\rangle_{V^{\prime}, V}+\left\langle\mathcal{A}_{a}(u(t)), w(t)-u(t)\right\rangle_{2, \infty}\right) d t \geq \int_{0}^{T}\langle\mathcal{H}(u(t)), w(t)-u(t)\rangle_{V^{\prime}, V^{\prime}} d t$ for every $w \in \mathcal{W}$, with $w(0)=u^{0}$, such that $w-u \in L^{\infty}\left([0, T] ; W^{2, \infty}(I)\right)$ and $\log \left(w_{x x}+a\right) \in$ $L^{1}\left([0, T] ; L^{1}(I)\right)$. This completes the proof of Theorem 1.2.

\section{ACKNOWLEDGMENTS}

The authors warmly thank the Center for Nonlinear Analysis (NSF Grants No. DMS0405343 and DMS-0635983), where part of this research was carried out. The research of I. Fonseca was partially funded by the National Science Foundation under Grant No. DMS0905778 and that of G. Leoni under Grant No. DMS-1007989. I. Fonseca and G. Leoni also acknowledge support of the National Science Foundation under the PIRE Grant No. OISE-0967140. The research of G. Dal Maso was also supported by the Italian Ministry of Education, University, and Research under the Project "Variational Problems with Multiple Scales" 2008 and by the European Research Council under Grant No. 290888 "Quasistatic and Dynamic Evolution Problems in Plasticity and Fracture".

\section{REFERENCES}

[1] Brezis H.: Opérateurs maximaux monotones et semi-groupes de contractions dans les espaces de Hilbert. North-Holland, Amsterdam-London; American Elsevier, New York, 1973.

[2] Butzer P.L., Nessel R.J.: Fourier analysis and approximation. Volume 1: one-dimensional theory. Academic Press, New York and London, 1971.

[3] Duport C. , Politi P. , and Villain J.: Growth instabilities induced by elasticity in a vicinal surface, $J$. Phys. I 5 (1995), 1317-1350.

[4] Fonseca I., Leoni G.: Modern methods in the calculus of variations: $L^{p}$ spaces. Springer Monographs in Mathematics. Springer, New York, 2007.

[5] Lions J.L.: Quelques méthodes de résolution des problèmes aux limites non linéaires. Dunod, GauthierVillars, Paris, 1969.

[6] Tersoff J., Phang Y. H. , Zhang Z. , and Lagally M. G.: Step-bunching instability of vicinal surfaces under stress, Phys. Rev. Lett. 75 (1995) 2730-2733.

[7] Xiang Y.: Derivation of a continuum model for epitaxial growth with elasticity on vicinal surface. SIAM J. Appl. Math. 63 (2002), 241-258.

[8] Xiang Y., E W.: Misfit elastic energy and a continuum model for epitaxial growth with elasticity on vicinal surfaces. Physical Review B 69 (2004), 035409-1-035409-16.

[9] Xu H., Xiang Y.: Derivation of a continuum model for the long-range elastic interaction on stepped epitaxial surfaces in 2+1 dimensions. SIAM J. Appl. Math. 69 (2009), no. 5, 1393-1414.

[10] Zeidler E.: Nonlinear functional analysis and its applications II/A: linear monotone operators. SpringerVerlag, New York, 1990.

[11] Zeidler E.: Nonlinear functional analysis and its applications II/B: nonlinear monotone operators. Springer-Verlag, New York, 1990

E-mail address, Gianni Dal Maso: dalmaso@sissa.it

E-mail address, Irene Fonseca: fonseca@andrew.cmu.edu

E-mail address, Giovanni Leoni: giovanni@andrew.cmu.edu

(G. Dal Maso) SISSA, Via Bonomea 265, 34136 Trieste, Italy

(I. Fonseca and G. Leoni) Carnegie Mellon University, Pittsburgh PA 15213-3890, USA 